\title{
Centrifuge testing of Dual Row Retaining Walls in Dry Sand: the influence of Earthquake Sequence and Multiple Flights
}

\author{
Srikanth S C Madabhushi ${ }^{* 1}$ and Stuart K Haigh ${ }^{\dagger 2}$ \\ ${ }^{1}$ University of California, Davis \\ ${ }^{2}$ University of Cambridge
}

\begin{abstract}
Multi-hazard threats from tsunami events preceded by large earthquakes have been put into sharp focus in recent times. Dual row retaining walls are soil-structure systems that can have large lateral capacity with a small horizontal extent, making them ideal for the next generation of coastal protection. However, the dynamics of their behaviour is a complex interaction problem. Centrifuge tests, with multiple earthquakes within a single flight as well as multiple flights with varied embedment ratio were conducted to elucidate the mechanics of these systems when founded in dry sand. The structural response shows permanent, plastic deformations during the early cycles dependent mainly on the PGA superposed with more elastic vibrations during prolonged shaking. The development of the soil stresses and stiffnesses mobilised is used to explain the overall system response. Finally, the recorded structural and soil behaviour during swing up and down are combined to show that the soil stress and strain state is effectively reset between flights. Overall, useful methods for judging the progressive response of a complex soil-structure system are presented which can help justify future comparisons between experimental datasets and understand the implications of practical dynamic design.
\end{abstract}

\section{Introduction}

Dual row retaining walls can be used as an efficient Tsunami defence in coastal regions. Two parallel rows of walls, connected by tie rods and with a soil infill can be erected to produce systems with a large lateral capacity but a relatively small horizontal extent. Such systems are currently being trialled in Kochi, Japan and the concept is illustrated in fig. 1.

The inclusion of a soil infill to provide strength and stiffness to the structure is an intriguing proposition. However, it is necessary to design for the large earthquake loading that will likely precede the tsunami and thus understand the complex dynamics of the dual row wall system.

To elucidate their behaviour a suite of centrifuge tests were conducted on these systems at the Schofield Centre at the University of Cambridge. Variations of the wall thickness, embedment to retained height ratio and wall spacing were studied. Though the dual row walls are envisaged as coastal defence structures, a set of tests concentrating on the behaviour in medium dense dry sands was also conducted to further the insight into their fundamental mechanical response. This insight can then be used to understand the results from more realistic test conditions, including cases with saturated and liquefiable foundation soil and the post-seismic performance of these systems subjected to Tsunami loading (Madabhushi and Haigh, 2018a; Madabhushi, 2018).

It is clearly desirable for cross comparisons to be made between these tests as part of the experimental parametric study. However, variations and assumptions made in the experimental set-up, test programme and analysis must be addressed. Practically, systems such as the dual row retaining walls founded in seismic regions may experience several moderate earthquakes within their design life. Multiple seismic events are

\footnotetext{
*sscmadabhushi@ucdavis.edu

†skh20@cam.ac.uk
} 
often applied during centrifuge testing which can usefully reveal the progressive response of different models. However the sequence in which they are applied is not necessarily fixed. Further, models are sometimes tested over multiple flights. In this paper, these two issues are explored through examination of the centrifuge data from selected tests on the dual row retaining walls. The system behaviour is better understood by considering separately the structural response, in terms of the forces developed in the tie rods and flexure of the walls, and the soil behaviour in terms of the mobilised stresses and stiffness. Based on the experiences from this testing, the implications for comparisons within the centrifuge suite described will be discussed in this paper. Further, it is envisaged that the wider conclusions will be useful for future dynamic centrifuge test programmes and data interpretation.

\section{Literature Review}

Kramer (1996) highlights the complexity of the dynamic response of even a single retaining wall, owing to the significant and time varying coupling between the wall movements and the soil stresses. Practical design methods have traditionally focussed on the limiting dynamic forces that can be generated given a representative peak ground acceleration, for example pseudo-static predictions based on the work of Okabe (1924) and Mononobe and Matsuo (1929). Limit equilibrium design calculations are conducted separately to predictions of the wall displacement (Newmark, 1965; Elms and Richards, 1979). Further, such methods do not attempt to account for the changing dynamic response as a result of the changing soil behaviour under cyclic loading, though many experimental and numerical studies have revealed their importance. For example, Whitman (1990) described the potential for increased soil pressures persisting after strong ground motion and which would alter the progressive response of structures in the field experiencing multiple earthquakes.

Zeng (1990) and Conti et al. (2012) also reported the results from dynamic centrifuge tests on different retaining structures and discussed the factors which influenced the cyclic response. In particular, the mechanisms by which the dynamic loading can increase the residual soil stresses and thus internal forces of the structural members are highlighted. Permanent wall displacements driven by the deformation or failure mechanisms of the soil can lead to larger soil stresses, for example as the soil strength is mobilised in the reacting passive regions. Equally, if the ground motion drives the soil relatively into the retaining system the soil stresses will be increased. This may occur for relatively heavy or stiff walls. Finally, cyclic loading can also result in densification of the soil mass and thus increases of the peak strength and stiffness. For the latter two phenomena the soil stresses acting on the wall may be increased without significant wall movement. Despite the different mechanisms, the greater soil pressures arising from the seismic loading can be broadly attributed to plastic straining of the soil mass.

\section{Centrifuge model details}

A centrifuge can be used to accurately replicate the dynamic behaviour of the full size dual row walls when using small scale models. The centrifugal acceleration increases the stresses in the model and thus the soil behaviour will be similar to the full scale field case. Schofield (1980) provides details of the $10 \mathrm{~m}$ diameter centrifuge testing facilities used.

In this work, the results from two centrifuge tests are discussed to assess the effect of multiple seismic events and flights. The primary difference between these two tests is that one featured stiff sheet pile walls and the other relatively flexible ones (Madabhushi and Haigh, 2016, 2018c). Simplified schematics of the models are illustrated in fig. 2, where the naming convention refers to the use of dry sand, the wall flexibility and the height:embedment:wall spacing ratio. Both models were subjected to $60 \mathrm{~g}$ at a point $\frac{1}{3}$ the height of the wall system. However, whilst the model with stiff piles was constructed inside a laminar box the more flexible walls were placed within a rigid box with a perspex front to the facilitate the use of the PIV technique (White et al., 2003). That a laminar box will minimise the boundary effects during shaking is well established (Brennan et al., 2006). In the rigid box, the extent to which the duxseal inserts can absorb the stress waves is reviewed and discussed by Campbell et al. (1991) or Coe et al. (1985). Further, Madabhushi and Haigh (2018b) note that the compliance of the soil and duxseal permits some lateral deflections which 
are comparable to those expected with laminar boundary conditions. Overall, the regions of interest within these models containers may be fairly compared.

In both cases, the wall systems were constructed at $1 g$ using S28 Hostun Sand (Azeiteiro et al., 2017). The sand was poured at $I_{d} \approx 80-85 \%$ using the automatic spot pluviator at the Schofield Centre (Madabhushi et al., 2006). The walls were placed once the pouring reached the base level, after which the pouring was continued inside and around them. The results from the instruments shown in fig. 2 are discussed in this paper, which includes piezoelectric accelerometers to measure the soil acceleration, load cells to measure the forces in the tie rod and full bridge strain gauges to measure the wall flexure. Additionally, tactile pressure sensing sheets manufactured by Tekscan are used to measure the horizontal effective stresses around the right wall. The Tekscan sheets were laminated and the remaining portions of the wall were covered in acetate to ensure a uniformly smooth interface between the wall and soil.

As indicated by fig. 2, each test had two flights, with the second flight of each model featuring a reduced ground level. This had the effect of increasing the retained height to embedded depth ratio. The ground level was reduced following the swinging up, shaking and swinging down of the initial model. The sand was carefully hoovered from outside the wall to create the desired model geometry and to avoid disturbance to the dense soil below. Some pertinent details of the prototype geometries are given in table 1. Unless stated otherwise, the results will be given at the prototype scale and the sign convention that leftward displacements are positive is used.

\section{Modelling multiple seismic events}

It is common in seismic centrifuge tests to subject the package to multiple shaking events. Table 2 details the characteristics of the earthquakes applied and the sequence in which they were fired for the tests discussed in this paper. Though similar earthquakes were applied, between the two tests the sequence of shaking was altered. In this section the effect of these multiple shakes on the dual row wall system is considered and the validity of cross comparisons from a single earthquake event between tests discussed.

From a geotechnical point of view, it is the onset of plasticity in the soil that implies that the order in which the earthquakes are fired can be significant. In selecting an earthquake sequence, it may be considered preferable that the tendency for plastic damage is greater in subsequent earthquakes. However, two complications are immediately apparent. The characteristics of the earthquake - be it the PGA, frequency content or number of cycles - that are predominantly responsible for generating plastic strains may be difficult to know 'a priori'. Measures such as the Arias Intensity (Arias, 1970) which try to capture the total energy content of the ground motion may be used but such integrated measures can miss the importance of the system response to specific frequencies or amplitude variations. Secondly, the development of plastic strains in one shaking event may in any case affect the deformation or failure mechanism of the subsequent event.

Overall, the evaluation of the system response to each shaking event ought to be considered in sequence and on a test by test basis. In this work, the different earthquakes were fired for various purposes. The small $1 \mathrm{~Hz}$ sine, with a PGA of $0.05 \mathrm{~g}$, was used as a non-destructive first motion in all tests mainly to help settle the model and try remove any unstable soil arches formed during the model construction. It was thought that this would help provide a more consistent starting stress state between tests, particularly owing to the complexity of inserting the dual row wall system and the automated pouring of the infill soil at $1 \mathrm{~g}$. The veracity of this assumption will be considered further in section 5. However, it is likely that dual row wall systems in the field - designed to withstand disaster level shaking events - would have experienced some smaller earthquakes beforehand which may also help justify this choice.

The response under larger cyclic loading was intended to elucidate the mechanics of the dual row wall vibration whilst the sine sweep was used to search for the natural frequency of the wall and soil system. Finally, the more realistic Kobe motion was used to judge the influence of asymmetric input motions.

Table 2 shows that the sine sweep preceded the large sine for the tests on the stiff wall system. To judge whether valid cross comparisons between centrifuge tests can be made, an assessment of the progressive dynamic responses of the dual row walls is required. 


\subsection{Measuring the system response}

Whilst the changing soil behaviour is challenging to measure directly, its effect on the structural response of the dual row retaining walls may be readily observed.

Figure 3 shows, over a number of shaking events, the generation of tensile forces in the ties between the walls for tests DF666 (fig. 3a) and DS666 (fig. 3b). In test DF666 the earthquakes were applied in sequence though a larger number of cycles were applied during EQ2. It is clear that whilst a small increase in tie force occurs during the initial shaking of EQ1 $(<5 \mathrm{kNm} / \mathrm{m})$, the most substantial increase occurs very quickly during EQ2. Further, this increase persists after the shaking ceases, though during the earthquake the tie force cycles about a larger mean value. This trend continues in subsequent earthquakes, with the same residual tie force maintained following the shaking.

In test DS666 the earthquake sequence is modified, with EQ3 preceding EQ2. The tie force increase is more gradual, in keeping with the slow build-up of accelerations during the sine sweep. After EQ3 finishes, the residual tie force is noticeably lower than the mean value during the shaking. The same residual value is returned to after the application of EQ2 and EQ4. Figure 3 gives strong evidence that shaking the dual wall systems initially increases the tie forces, but that there is a limit to this increase and subsequent shakes only result in 'elastic' (in the sense they are recovered) variations on top of this. This could suggest that despite the different stress paths, there is a limit to the plastic strains that can be generated across the soil body in the dual wall system. Continued shaking thereafter (with different frequency contents but similar maximum PGA's) only induces elastic stress changes in the soil. The distinction between soil strains induced by the dynamic and permanent wall displacements, from the soil being driven into the walls and those from rearrangement and densification of the soil mass (Conti et al., 2012) will be discussed further in section 4.2.3.

It is desirable to compare the results from DF666 and DS666 during EQ2. The evidence from fig. 3 firstly suggests that the dynamic variations in the tie force from later cycles may be readily compared. The more subtle point is whether the mean value that these oscillations occur about may also be compared. For fig. 3b, the maximum PGA in EQ3 is similar to EQ2 and the mean dynamic value stops increasing before the shaking does. On this evidence it can be argued that the mean dynamic value observed for EQ2 is similar to that which would have been generated even if EQ3 had not been applied. Therefore, the total tie force may be compared between these two tests.

The residual tie force of $64 \mathrm{kNm} / \mathrm{m}$ between the stiff walls is larger than the $31 \mathrm{kNm} / \mathrm{m}$ between the flexible walls. The values are roughly in keeping with the initial ratio of tie forces before the large magnitude shaking and indicates a larger tie force is required to equilibrate the stiff walls. Figure 4 illustrates the potentially different distributions of soil stresses around a single tied flexible and stiff wall, or equivalently half of a dual row system, which could justify this difference in tie force. The tendency of tied flexible walls to bow outwards can result in greater mobilisation of soil stresses near the surface which can move the point of action of the reacting forces upwards. By contrast, a stiffer tied wall more rigidly rotates about the tie and may mobilise a more uniform proportion of the passive soil strength. As shown by fig. 4, the lower point of action in this case requires a greater tensile force in the tie to equilibrate the overturning moment. The amplitude of the dynamic variations of tie force is also much larger for the stiff walls. This suggests that it is harder for the two stiffer walls to move together under dynamic loading.

In a similar vein, fig. 5 presents the measured wall displacements and the response of selected strain gauges for the same tests. The leftward permanent displacement developed in DF666, discussed in greater detail by Madabhushi and Haigh (2018c), is clearly illustrated by fig. 5a. Again, these appear to develop very early during EQ2 and subsequent shaking only results in oscillations about this value. The increase in the bending moment follows the same trend and the same conclusions are generally true for the bending in test DS666 (fig. 5b), with the constant mean bending value reached mid way through EQ3 in this case. However, the effect of the asymmetric Kobe motion is shown to produce relatively larger permanent displacements of the stiff dual wall system. Interestingly, that this change does not manifest in the measured residual wall bending or the tie force may indicate that the walls have either suffered rigid body translation or rotation, i.e only the constants of integration for the displacement from integrating the bending moment have changed. The measured permanent displacement of $0.075 \mathrm{~m}$ following the Kobe motion could imply at most a rotation of $0.9^{\circ}$ about the ground surface or a smaller rotation with some translation. These wall movements may not induce large shear or normal strains in the soil. This could leave the mean soil stresses unchanged, consistent with the unchanged residual internal forces of the structural elements. 
Overall, the consideration of the system response in this way also gives an insight into the characteristics of the ground motions which affect the structural demands on the system. Given a system far from resonance, design methods which concentrate on the PGA of the input may be appropriate for estimating the peak dynamic force demands on a system. However, subtleties such as the sensitivity to asymmetric shaking or predicting the progressive accumulation of damage, particularly displacements, would be less readily predicted by such simplified methods. The structural demands on the systems static equilibrium and during the dynamic loading were also shown to depend on the wall flexibility. This could necessitate an optimisation in the design process as the wall stiffness affects both the loads and the resulting behaviour.

\subsection{Measuring the soil response}

\subsubsection{Horizontal Earth Pressures}

The horizontal effective stresses around the dual row walls arguably gives a more direct measure of the effect of multiple seismic events. However, the ability to reliably measure dynamic earth pressures with tactile pressure sensors is still relatively new in centrifuge testing; careful calibration and interpretation of the results is required (Paikowsky and Hajduk, 1997; Dashti et al., 2012; Madabhushi and Haigh, 2018d).

Examples of the time varying horizontal effective stresses measured on the internal and external faces of the right wall are plotted in fig. 6 . To verify internal consistency, the earth pressure distributions were double integrated and compared with the bending moments from the the strain gauges. The traces plotted in fig. 6 represent only the times where good matches were obtained, as previously demonstrated by Madabhushi and Haigh (2018d). It was observed that during the end of some shaking events the change in mean value from the tactile pressure sensing sheets implied bending moment changes inconsistent with the strain gauge results. Further study is required to determine whether this is a physical phenomenon or an artefact of rapid cyclic loading on the Tekscan sheets.

During the shaking, the earth pressure variation inside and outside the walls are in keeping with the discussed trends. Figure 6a shows that following EQ2 the earth pressures are increased both inside and outside the wall, and previous results indicate this requires greater tie force to maintain equilibrium and that larger bending moments are generated. Figure $6 \mathrm{~b}$ shows a steady increase of the effective stresses during EQ3.

Conceptually, the static stresses can increase for the external soil if the wall vibration results in wall movements relatively into the external soil which would increase the mobilised passive earth pressure coefficient. However, that the internal static soil pressures should simultaneously rise means changes to the mobilised earth pressure coefficients from wall movements alone may not be sufficient to explain the observed behaviour. Remembering the symmetry of the dual row wall construction, during the dynamic displacement in a given direction the leading wall movements will be restrained by both the external soil and the lagging wall dragged into the infill via the tie rod. As a result, the ground motion may result in the infill soil being relatively driven into the leading wall and thus increasing the soil stresses. It is also known that vibration of the medium dense sand could induce densification, i.e a decrease of voids ratio, both inside and outside the walls. Whilst clearly the total mass of sand is fixed and thus the vertical stresses will not increase (in fact at a specific wall height the vertical stress would decrease if the soil relatively settles) an increased horizontal stress is plausible. Micro-mechanically, as the grains try to settle into the existing voids they will act to push nearby grains away. This may also have the effect of increasing the lateral stress even without the walls suffering permanent displacement.

\subsubsection{Cyclic Shear Response}

Monotonically shearing a soil leads to a degradation of the shear modulus. However, large cyclic shear strains applied over many cycles to sands can lead to an increase of shear modulus under drained conditions. This is often attributed to shear induced volumetric strains (Dobry and Vucetic, 1987; Kramer, 1996). The mean confining pressure can also shift the modulus reduction curves; larger mean confining pressures give slower degradation with cyclic shear strain, particularly for low plasticity sandy soils (Isao and Ishibashi, 1992).

Section 4.1 illustrated that permanent wall displacements were generated, particularly for case DF666. Meanwhile, in section 4.2 the increase of horizontal effective stress justified the locked-in bending moments and tie forces observed, but also suggests the mean confining pressure in the soil is increased. The effect on 
the soil stiffness can be abstracted from the measurements of the soil accelerations. The relative accelerations along an instrumented 'soil column' can be used to infer the cyclic shear stress whilst the relative displacement, obtained from the double integral of the accelerations can give an average cyclic shear strain (Brennan et al., 2005). That constant shear stresses and monotonic increases of shear strain are not registered should be borne in mind.

Figure 7 shows the cyclic shear stress-strain loops across multiple shaking events for both test DF666 and DS666 from accelerometers between and outside the dual row walls. The accelerations were bandpass filtered at $0.2 \%$ and $90 \%$ of the Nyquist frequency. The progression of the average secant stiffness per cycle is also plotted. Azeiteiro et al. (2017) present the results from Bender element tests on Hostun sand at a very similar initial relative density and plot the variation of $G_{\max }$ with $p^{\prime}$. For soil at the mid-depth of the infill the mean confining pressure could vary between $50-220 \mathrm{kPa}$ for horizontal stress variations between $K_{A}-K_{P}$. This implies limiting small strain shear stiffness's between $75-130 \mathrm{MPa}$ for soil at this voids ratio.

The results from DF666 in fig. 7a show the soil response outside the left wall is quite different to the response between the two walls. By EQ2, the soil outside the walls reaches a low mobilised stiffness which is fairly constant during the subsequent cycling. By contrast, the soil between the walls' stiffness shows a large increase (though remains less than the maximum $G_{\max }$ calculated previously) as the soil is cyclically loaded. The larger shear stresses experienced by the infill can be attributed to the amplification of the accelerations as they propagate through the wall-soil system (Madabhushi and Haigh, 2018c). Whilst the rapid increase of average stiffness towards the end of EQ2 may be attributed to a reduction in the applied stresses, there is clear evidence of an increased secant stiffness during the earlier, constant magnitude stress cycles. Volumetric strains in the soil as well as an increase of mean confining pressure have been raised as possible mechanisms that can lead to increases of the mobilised soil stiffness. This increased stiffness persists during events EQ3a and EQ3b.

On the other hand, fig. 7b reveals that the infill soil in system DS666 doesn't experience a stiffening effect. The soil response outside and between the walls is quite similar and shows a degradation to a plateau during EQ3 which remains mobilised during EQ2. Interestingly, the maximum cyclic strains are larger than developed for DF666. In both tests the soil's starting state will be comparable, and thus the strong interaction between the wall behaviour and the utilisation of the soil strength and stiffness is highlighted. Potentially, the limited flexure of the stiffer walls leads to less volumetric straining of the infill whilst the rigid body displacements are larger.

In section 4.1, the results from system DF666 showed the most significant changes (i.e increased mean tie force, bending moments and wall displacement) occurred during the early cycles of EQ2 after which most measurements exhibited a more 'steady-state' response. The horizontal stresses in section 4.2 were largely in keeping with this observation. Due to reversed motion sequence, the measurements from DS666 showed a gradual increase during EQ3 with the final mean value returned to after EQ2. The inferred changes of soil stiffness are consistent in explaining the distinct behaviour from the two tests. The source of the increased soil stiffness in DF666 is now explored.

\subsubsection{Displacements and Strains}

The accumulation of soil and wall displacements during test DF666 can be obtained by using PIV. Figures 8a to 8b shows the tracked deformations following swing up of the model and after the application of EQ1. By the end of EQ1 the total displacements are minimal and primarily due to the vertical settlement following the model swing up. By contrast, at the end of EQ2 fig. 8c shows significant permanent displacements. The walls have displaced leftwards overall but also visibly bowed out. The soil infill thus shows lateral displacements but also a larger amount of vertical settlement.

The soil deformations were tracked during EQ1 and EQ2. An estimate of the 1D compressive strain along the infill centreline is shown in fig. 9 - calculated using the incremental vertical displacements at this location. Figure 9 reveals a number of interesting trends. The minimal strain generated during EQ1 is expected. The strains cumulate along the infill height with the stronger and stiffer soil at greater depth and thus with a larger confining pressure straining the least. This trend is maintained during EQ2 though larger strains are calculated. Further, the changing rate of development of the strain is also showcased. During the initial cycling the strain increases rapidly, in line with the previous observations of the large 
dynamic variations during the initial 'transient' phase. Following this fig. 9 reveals a slower but discernible accumulation of strain, particularly near the top of the infill. This accumulation of strain helps justify the prolonged variation of soil stiffness seen for test DF666. That it arises from the outward bowing of the flexible walls can also explain the lack of this stiffening effect for the stiff walls in test DS666. Figure 6 shows that in both tests the horizontal effective stresses increase during the cyclic loading. It may be concluded that the stiffening effect from the volumetric strain is more dominant than that from the locked-in stresses increasing the mean confining pressure.

The average volumetric strain across the infill can also be calculated from the measured wall displacement and surface soil settlement. Between the beginning and end of EQ2, the total infill volume is calculated to have reduced by $\approx 0.7 \%$; the effect of the soil settlement outweighs the outward bowing of the flexible walls. Overall, an average volumetric strain of $0.7 \%$ implies a densification of $\approx 2.5 \%$ in terms of relative density, though fig. 9 shows that the densification will not be uniform across the infill height.

The subtlety of the soil behaviour has many implications in terms of the effectiveness of the dual row system as a coastal defence. This is particularly true as both the system strength and stiffness are seen to change during prolonged cyclic loading. As mentioned previously, during design, simplifications of the input motion can be appropriate when estimating the total loads and associated structural requirements. However, when assessing the dual row walls' resilience there are not currently straightforward methods to account for the potential shear induced volumetric strains and the associated soil stiffening, particularly as this depends on the dynamic interaction between the soil and walls.

\section{Modelling different embedments with multiple flights}

The degree to which multiple flights of the same model on a centrifuge can be considered independent tests is difficult to quantify and potentially quite problem specific. In this work, following swing down the external ground level was lowered via vacuuming to minimise the disturbance to the dense soil for the subsequent tests. In general, lowering the embedment ratio of the wall system should result in a decreased factor of safety, and thus increased wall displacements, bending and ultimately soil stresses and strains. However, section 4.2 clearly demonstrates the generation of plastic stresses and strains in the soil as a consequence of the large earthquake loading. This may affect the stress states that subsequent tests can reach. Therefore, it is necessary to consider whether the stress and strain state are effectively 'reset' by the swinging down of the centrifuge model.

Clearly, as the centrifugal accelerations are reduced and the model swings down the soil is unloaded significantly. In general, the soil should follow the unload-line and thus without experiencing greater stresses will remain stiffer than the initially tested soil. It was also shown in section 4.2 that greater lateral earth pressure coefficients are mobilised as a result of the shaking. Ignoring stress dependent dilation and assuming the wall is rigid relative to the soil; for geometrically similar retaining walls in sand (i.e. with different total heights but the same ratio of retained to embedded height) the mobilised earth pressure coefficients will be congruent. Thus, for a centrifuge model subjected to varying centrifugal accelerations, during swing up say, the mobilised earth pressure coefficients should also not change under the same assumptions. Therefore, the subsequent static equilibrium when the sand is vacuumed and the model swung up again would be affected if the previously experienced shaking results in locked in stresses.

Simpson and Powrie (2001) summarise methods to assess the relative flexibility of a retaining wall, including the flexibility number, $F$ derived by Li (1990) and given in eq. (1).

$$
F=\frac{G^{*} H^{4}}{E I}
$$

Equation (1) quantifies the ratio of wall bending to rigid body rotation for a propped wall, where $H$ is the total height, $E I$ is the flexural stiffness and $G^{*}$ is the rate of increase of the shear modulus. The derivation uses the average rotation of a rigid wall to enforce equilibrium by assuming elastic decreases and increases on the active and passive side respectively. The use of $G^{*}$ to characterise the soil stiffness will have a dependence on the mean confining pressure $p^{\prime}$ which may be expressed by eq. (2), where a power law with constants $\alpha$ and $\beta$ is used.

$$
G^{*} \propto G=\alpha\left(p^{\prime}\right)^{\beta}
$$


Though clearly simplified, eq. (1) and eq. (2) can be used to show the approximate change of relative wall flexibility of a retaining wall in a centrifuge model with $g$ level. At $N \times g$, eq. (3) gives the ratio with respect to the initial flexibility ratio of the model wall at $1 \mathrm{~g}$.

$$
\frac{F_{N g}}{F_{1 g}}=\frac{\frac{\alpha\left(N p^{\prime}\right)^{\beta}(N H)^{4}}{E I N^{4}}}{\frac{\alpha\left(p^{\prime}\right)^{\beta} H^{4}}{E I}}=N^{\beta}
$$

The value of $\beta$ would typically be between $0.5-1.0$ depending on the strain level (?), and thus eq. (3) shows that as a model retaining wall is swung up in a centrifuge its flexibility number increases due to the change of soil stiffness. Li (1990) proposed eq. (1) as a binary divide between stiff and flexible walls with the transition defined when the deflection at the excavation depth equals the toe deflection. In principle, a centrifuge model of a rigid wall should ensure this critical value is not exceeded at the final $g$ level. For tests on flexible walls, in most cases the system will start as a rigid wall at low $g$ levels before transitioning to a flexible wall during swing up. If the wall is permitted to deflect during swing up (i.e not supported by any additional props) it may be important to consider whether the final static equilibrium is independent of the preceding $g$ levels. The situation is further complicated by the varying mobilisation of the soil stiffness with strain and thus deflections of the wall - particularly if the displacement mode is expected to change during swing up.

Each wall of the dual row system is similar to a single wall with a rigid prop. The experimental results can thus be used to explore the above discussion. Figure 10a shows the distribution of bending moments during the swing up stages of DF666 and after the previously described 'non-destructive' EQ1, normalised with the applied centrifugal accelerations. It is immediately apparent that during swing up the distribution of normalised bending moments continually changes with the increasing $g$ level. That the distributions are not coincident demonstrates that the model retaining wall at different $g$ levels are not mathematically similar.

The earth pressures recorded and calculated lateral earth pressure coefficients (assuming smooth walls and thus linearly increasing vertical stresses) are plotted in fig. 10b. It is clearly shown that the stresses increase with increasing $g$ level. The inferred lateral earth pressure coefficients, which normalise for the increase of soil weight, are consistent with the bending moments in showing the changing retaining wall behaviour as it effectively increases in total height. The equivalent mobilised friction angles are also indicated.

As a brief aside, the effect of EQ1 on the earth pressures and bending moment is demonstrated by fig. 10. The bending moments are slightly increased whilst there is a clear increase of the earth pressures, particularly within the infill. The increase of the bending moment gradient near the top of the walls, as well as fig. 3a previously, show that the tie forces also show a small permanent increase. The benefit of this small shake in producing a smoother, more continuous bending moment and earth pressure distribution is exemplified. Within the infill, arching of the soil between the walls may occur due to the pluviation method within the narrow gap and thus alter the vertical stress distribution. This is more a consequence of the system construction at model scale and is considered an unrealistic starting stress state for the dual row retaining walls.

The lack of similarity during swing down is also shown in fig. 10a. As the $g$ level during swing down was not known, the gauge responses were normalised with the values at $60 \mathrm{~g}$ and the lack of coincidence between the individual gauges indicates that the bending moment distribution is changing. Overall, the swing up and swing down process results in a build up and dissipation of strain energy as the walls' curvature is increased and decreased. Whether this change acts to remove the locked in earth pressure coefficients or not is clarified by fig. 10b. Whilst the lateral effective stresses clearly decrease, the earth pressure coefficients at $1 g$ are larger than those before the test illustrating that the soil retains at least partial memory of the shaking.

Following this, the sand was vacuumed before the model was swung up again to commence test DF846. Approximately $33 \mathrm{~mm}$ of sand was removed from the external soil (i.e $2 \mathrm{~m}$ prototype), and fig. 10b shows that this is a large portion of the soil that retained the larger earth pressure coefficient. Figure 11 presents an equivalent distribution for test DF846. The first point to note is that the distribution at $1 \mathrm{~g}$ is altered relative to the $1 \mathrm{~g}$ end state of DF666. In general, the lateral earth pressure coefficient is reduced relative to DF666 which indicates that the soil has 'relaxed' following the vacuuming. This adds weight to the independence of DF846 from the previous test. The larger coefficient near the surface is perhaps more a result of the new equilibrium requirements of the effectively taller wall. The implied mobilisation of large friction angles at low effective stresses may be partly rationalised by soil dilation but the reduced signal-to-noise ratio at lower 
$g$ levels and uncertainty of the vertical stress distribution should also be borne in mind.

Whilst fig. 11 provides evidence that the new equilibrium of the wall is independent of the previous test and shaking, fig. 12 allows memory of the previous tests soil densification and stiffness increase to be judged. The evolution of the soil stiffness implied by the calculated dynamic stress-strain loops is plotted for both tests DF846 and DS846. Figure 12a exhibits very similar trends to fig. 7a - with a clear increase of the soil stiffness observed between the flexible walls. Further, the initial values during EQ2 of DF846 are lower than the final values from EQ3b of DF666, despite the similar maximum accelerations applied. Between fig. $12 \mathrm{~b}$ and fig. $7 \mathrm{~b}$ the soil stiffness also appears to have been effectively reset by the model swing down and lowering of the embedment depth. The previously observed volumetric strains between the flexible walls may suggest a subsequent centrifuge flight should begin stiffer due to the soil densification. However, the decrease of relative flexibility of the wall with respect to the soil during swing down could be important. Figure 10a showed the decrease of the bending moment and thus release of the strain energy in the walls during swing down. As the wall response becomes 'stiffer' and they try to straighten, the soil could be sheared and experience a volume change. In particular, during swing down the mean confining pressure will drop and thus the soil will more readily dilate. This could offset the shear induced volumetric strains from the dynamic testing and contribute towards the 'resetting' of stress and strain state observed in the next flight.

\section{Conclusions}

Overall, the effect of subjecting the model dual row retaining walls to multiple seismic events has been considered in the context of the response of two tests on flexible and stiff walls, DF666 and DS666. Between these two tests the earthquake sequence differs and the nature of the earthquakes, in terms of number of cycles, also varied. The measured structural response of the system, i.e. the tie forces or wall bending, suggests that the part of the model that goes plastic does so in a small number of large-amplitude cycles and subsequent shaking produces only elastic vibrations. The residual static values seemed to depend more on the largest accelerations that were applied than the number of cycles or frequency. This plateau of plastic straining in the system validates the cross comparison of total values from the same earthquake, applied in different sequences, between these tests. Potentially, simplifications during seismic design that focus on the PGA to estimate the total dynamic loads are also partially supported by these findings, particularly if the system's natural frequencies are known to lie outside the expected loading range.

Examination of the dynamic response of the soil shows that horizontal stresses both inside and outside the walls can be locked-in by the applied earthquakes. Whilst this may contribute towards soil hardening, the cyclic shear responses abstracted from both tests reveals that the soil generally softens in DS666. In DF666 however, there is a clear trend of increased soil stiffness between the walls during the constant magnitude cycling, which agrees with the diminished dynamic response of the system recorded. The deformation mechanism of the flexible dual row walls allows the accumulation of volumetric strains in the soil which can also lead to stiffening of the soil, and which logically would be more limited between the stiffer walls. From a coastal defence perspective, the potential increase of the system stiffness during prolonged ground motions results in a reduced accumulation of permanent displacements and is indicative of an efficient and robust system. That the relative flexibility of the dual row walls promotes this greater utilisation of the soils plasticity may be an important design consideration.

Overall, for valid comparisons to be made between centrifuge tests with different earthquake sequences evidence of limited permanent forces and elastic dynamic oscillations should be sought. In terms of comparisons of displacements, the potential for both increases and decreases of the soils' contribution towards to system stiffness should be considered and experimentally tracked if possible. That predictions of these changes in complex interaction problems are not readily calculated for practical design should be borne in mind, though the potential benefits in terms of a designs resilience also not neglected.

The use of multiple centrifuge flights with the same model was also considered. For retaining wall models, the increased $g$ level leads to an increase of the wall's flexibility relative to the soil, which was evidenced by the combined bending moment and soil pressure data showing the changing soil-structure interaction as a function of the $g$ level. The usefulness of a small, non-destructive motion to provide a more consistent starting stress state between tests was also shown. An investigation of the horizontal stresses supports the 
independence between the equilibrium of two centrifuge tests on the same model where the retained height was increased by vacuuming. The argument can also be cast in terms of the relaxation of the mobilised earth pressure coefficients but the uncertainty of the vertical stress distribution should be borne in mind. Though the seismic loading led to observable increases of soil stiffness in the first flight, this was seemingly reset in the subsequent flight. The potential for volumetric straining of the soil as the walls become relatively stiffer and the soil more dilative during swing down could justify this. In closing, the importance of the swing up and swing down data in tracking the soil stress and strain state in complex soil structure interaction problems is highlighted, and should be considered when comparing experimental data with field observations and advising practical design.

\section{References}

Arias, A. (1970). A measure of earthquake intensity, Seismic Design for Nuclear Power Plants, Cambridge, Mass. Massachusetts Inst. of Tech. Press, pp. 438-483.

Azeiteiro, R. J. N., Coelho, P. A. L. F., Taborda, D. M. G. and Grazina, J. C. D. (2017). Critical State-Based Interpretation of the Monotonic Behavior of Hostun Sand, Journal of Geotechnical and Geoenvironmental Engineering 143(5): 1-14.

Brennan, A. J., Madabhushi, S. P. G. and Houghton, N. (2006). Comparing laminar and equivalent shear beam (ESB) containers for dynamic centrifuge modelling, in C. W. W. Ng, L. M. Zhang and Y. H. Wang (eds), Physical modelling in geotechnics : 6th ICPMG '06, Vol. 1-2 of Balkema-proceedings and monographs in engineering, water and earth sciences, Taylor \& Francis, United Kingdom, pp. 171-176.

Brennan, A. J., Thusyanthan, N. . I. and Madabhushi, S. P. G. (2005). Evaluation of Shear Modulus and Damping in Dynamic Centrifuge Tests, Journal of Geotechnical and Geoenvironmental Engineering 131(12): 1488-1497.

Campbell, D. J., Cheney, J. A. and Kutter, B. L. (1991). Boundary effects in dynamic centrifuge model tests, Centrifuge 1991 : proceedings of the International Conference Centrifuge 1991, A.A. Balkema, Boulder, Colorado, pp. 441-448.

Coe, C. J., Prevost, J. H. and Scanlan, R. H. (1985). Dynamic stress wave reflections/attenuation: Earthquake simulation in centrifuge soil models, Earthquake Engineering \& Structural Dynamics 13(1): 109-128.

Conti, R., Madabhushi, S. P. G. and Viggiani, G. M. B. (2012). On the behaviour of flexible retaining walls under seismic actions, Géotechnique (12): 1-14.

Dashti, S., Gillis, K. and Ghayoomi, M. (2012). Sensing of Lateral Seismic Earth Pressures in Geotechnical Centrifuge Models, 15th World Conference on Earthquake Engineering, Lisbon Portugal, Sociedade Portuguesa de Engenharia Sismica (SPES), pp. 1-10.

Dobry, R. and Vucetic, M. (1987). Dynamic properties and seismic response of soft clay deposits, The International Symposium on Geotechnical Engineering of Soft Soils, pp. 51-87.

Elms, D. G. and Richards, R. (1979). Seismic Design of Gravity Retaining Walls, Bulletin of the New Zealand Nat. Soc. for Earthquake Engineering 12(2): 114-121.

Giken (2016). Structural Revolution of Disaster Prevention Infrastructure using Implant Structure.

Isao, I. and Ishibashi, I. (1992). Discussion of "Effect of Soil Plasticity on Cyclic Response" by M. Vucetic and R. Dobry, Journal of Geotechnical Engineering 118(5): 830-832.

Kramer, S. L. (1996). Geotechnical Earthquake Engineering, Prentice Hall.

Li, S. F. (1990). On the analysis of singly-propped diaphragm walls, $\mathrm{PhD}$ thesis, King's College London. 
Madabhushi, S. P. G., Houghton, N. E. and Haigh, S. K. (2006). A new automatic sand pourer for model preparation at University of Cambridge, Proceedings of the Sixth International Conference on Physical Modelling in Geotechnics, Taylor \& Francis, Hong Kong, pp. 217-222.

Madabhushi, S. S. C. (2018). Multi-Hazard Modelling of Dual Row Retaining Walls, Phd thesis, University of Cambridge.

Madabhushi, S. S. C. and Haigh, S. K. (2016). The Influence of Embedment on the Seismic Performance of Dual Row Retaining Walls, 6th International Conference on Recent Advances in Geotechnical Earthquake Engineering and Soil Dynamics, Missouri University of Science and Technology, New Delhi, India.

Madabhushi, S. S. C. and Haigh, S. K. (2018a). A Comparison between the Dynamic Behaviour of Flexible Dual Row Walls Founded in Dry and Liquefiable Sands, 1st International Conference on Press-in Engineering, pp. 265-272.

Madabhushi, S. S. C. and Haigh, S. K. (2018b). Combining Experimental and Numerical Methods to investigate the seismic performance of dual row retaining walls in Dry Sand, 16th European Conference on Earthquake Engineering.

Madabhushi, S. S. C. and Haigh, S. K. (2018c). Measuring the behaviour of dual row retaining walls in dry sands using centrifuge tests, International Conference of Physical Modelling in Geotechnics, London, UK, pp. 639-644.

Madabhushi, S. S. C. and Haigh, S. K. (2018d). Using Tactile Pressure Sensors to Measure Dynamic Earth Pressures around Dual Row Walls, International Journal of Physical Modelling in Geotechnics ((Ahead of print)).

Mononobe, N. and Matsuo, H. (1929). On determination of earth pressure during earthquake, Proceedings of the World Engineering Congress, Tokyo, Vol. 9, Kogakkai, Tokyo, Japan, p. 176.

Newmark, N. M. (1965). Effects of Earthquakes on Dams and Embankments, Géotechnique 15(2): 139-160.

Okabe, S. (1924). General Theory on Earth Pressure and Seismic Stability of Retaining Wall and Dam, Journal of Japan Society of Civil Engineers 10(6): 1277-1323.

Paikowsky, S. G. and Hajduk, E. L. (1997). Calibration Use of Grid-Based Tactile Pressure Sensors in Granular Material, Geotechnical Testing Journal 20(2): 218-241.

Schofield, A. N. (1980). Cambridge Geotechnical Centrifuge Operations, Géotechnique 30(3): 227-268.

Simpson, B. and Powrie, W. (2001). Embedded retaining walls: theory, practice and understanding, Proceedings of the 15th International Conference on Soil Mechanics and Geotechnical Engineering pp. pp. $2505-2524$.

White, D. J., Take, W. A. and Bolton, M. D. (2003). Soil deformation measurement using particle image velocimetry (PIV) and photogrammetry, Géotechnique 53(7): 619-631.

Whitman, R. V. (1990). Seismic design and behavior of gravity retaining walls, Proc. of ASCE Specialty Conference on Design and Performance of Earth Retaining Structures, pp. 817-834.

Zeng, X. (1990). Modelling the behaviour of quay walls in earthquakes, Phd thesis, University of Cambridge. 


\section{List of Figures}

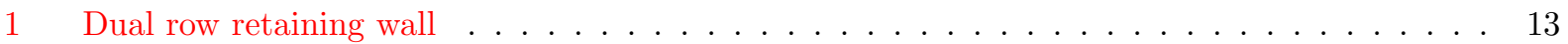

2 Simplified centrifuge model schematics . . . . . . . . . . . . . . . . . . . . . . . . 14

3 Generation of tie forces over multiple earthquakes . . . . . . . . . . . . . . . 15

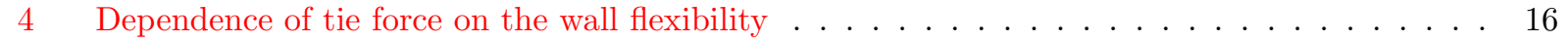

5 Generation of displacements and bending moments over multiple earthquakes . . . . . . . 17

6 Generation of soil pressures over multiple earthquakes . . . . . . . . . . . . . . 18

$7 \quad$ Dynamic shear stress-strain plots over multiple earthquakes . . . . . . . . . . . . . . 19

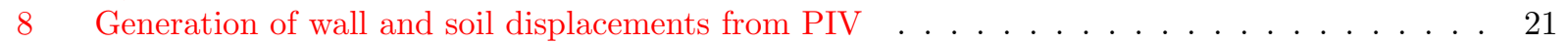

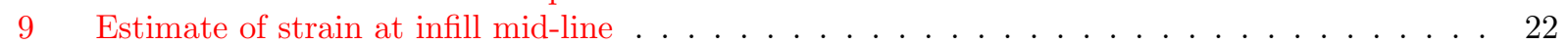

10 Dissimilarity of wall behaviour during swing up and swing down, DF666 . . . . . . . . 23

11 Earth pressure development in a previously shaken but subsequently hoovered model, DF846 24

12 Inferred average stiffness in a second centrifuge flight from dynamic stress strain loops . . . . 25 


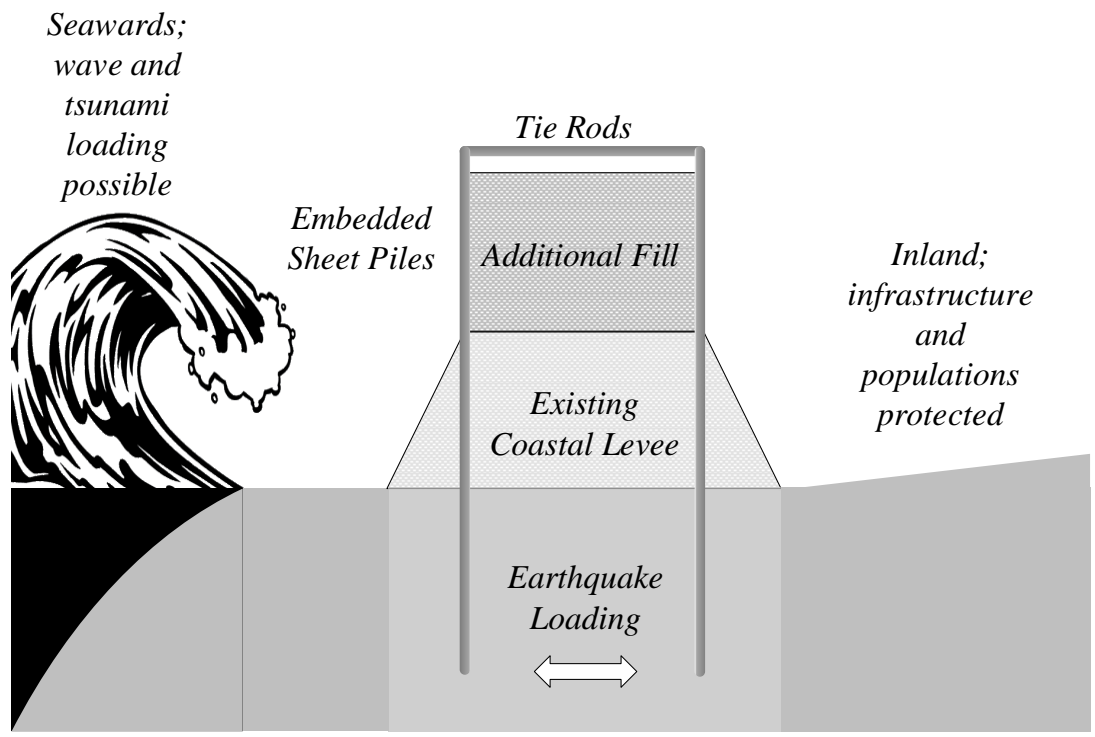

(a) Conceptual illustration, following Madabhushi and Haigh (2016)

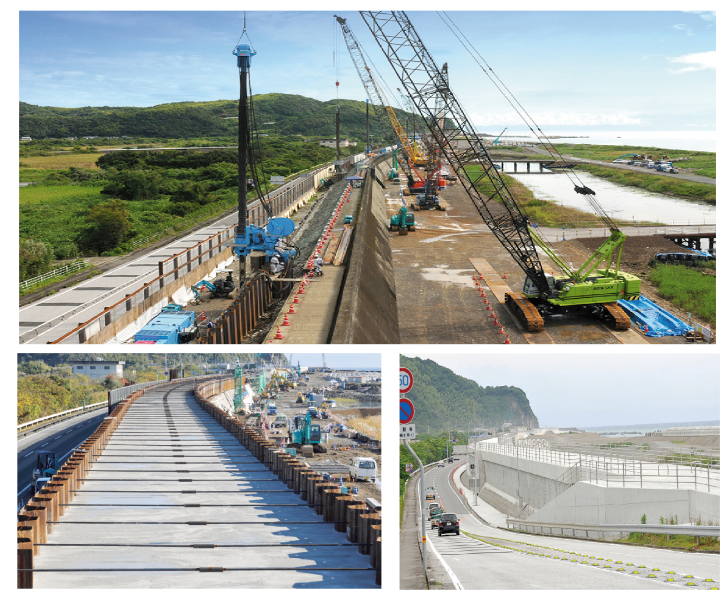

(b) Photos of dual row retaining wall (Giken, 2016)

Figure 1: Dual row retaining wall 


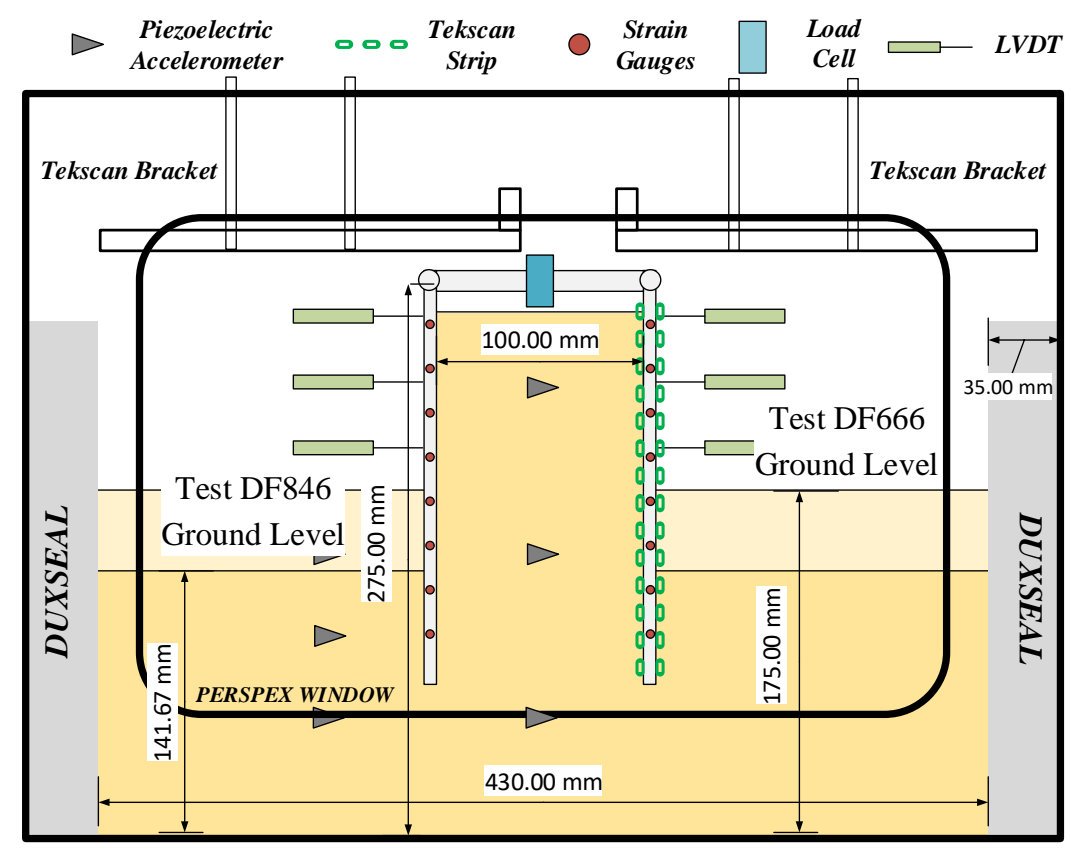

(a) DF666 \& DF846

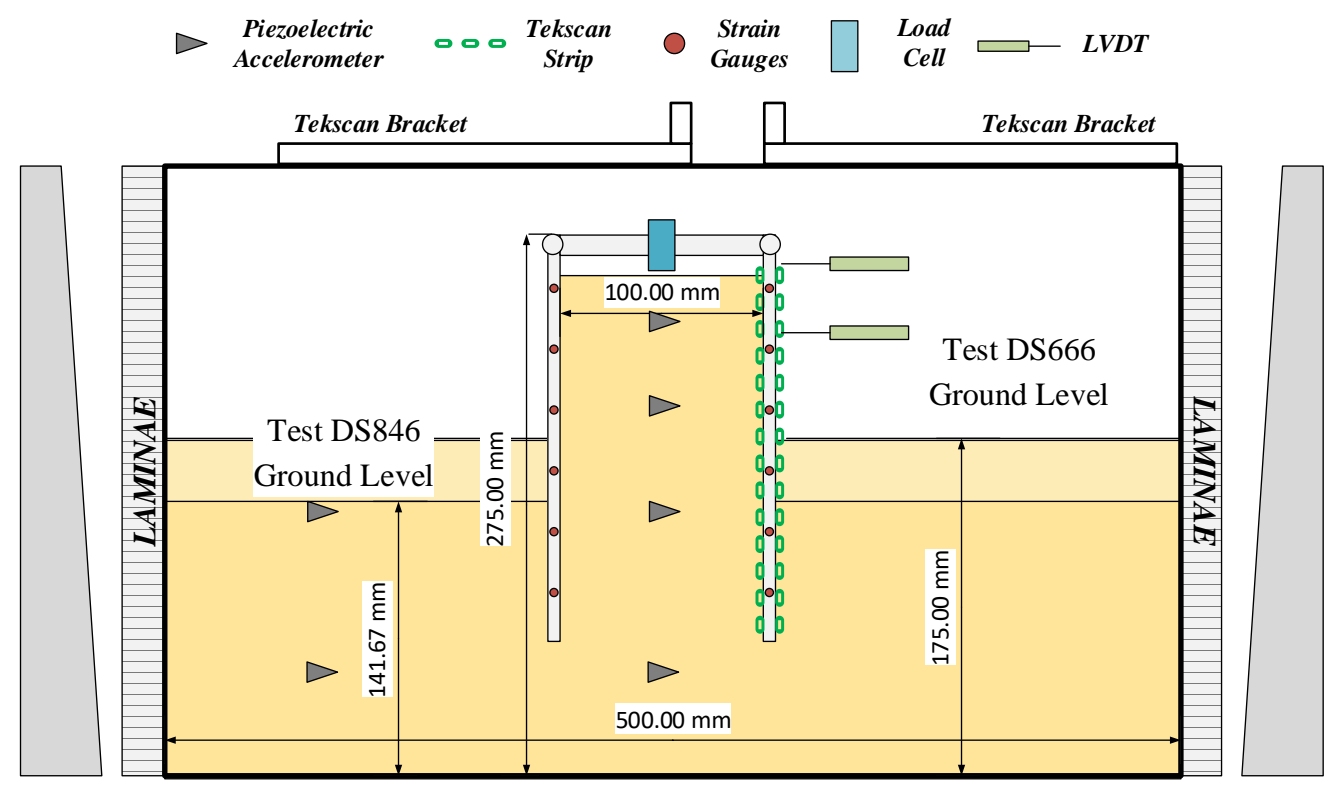

(b) DS666 \& DS846

Figure 2: Simplified centrifuge model schematics 

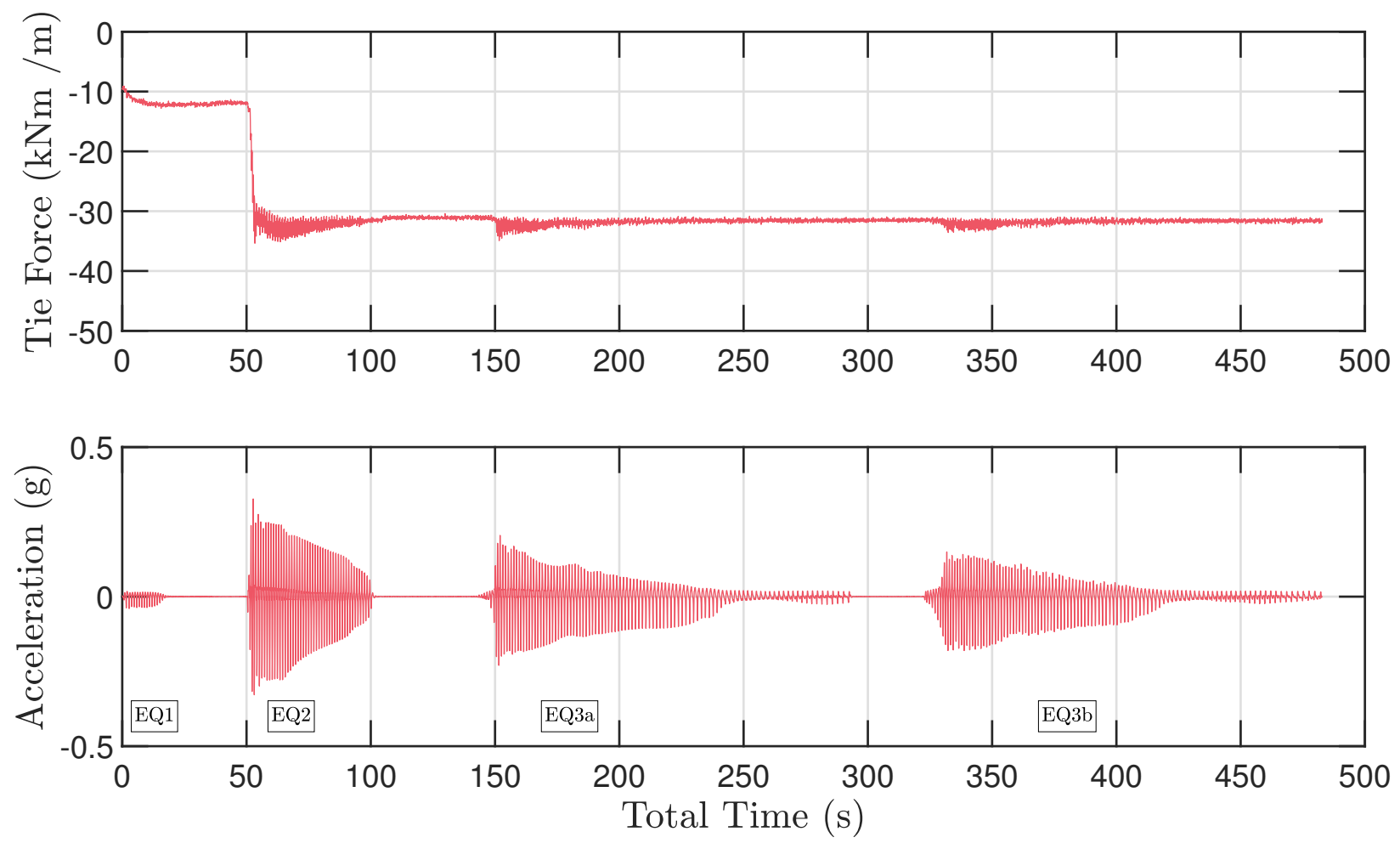

(a) All earthquakes, DF666
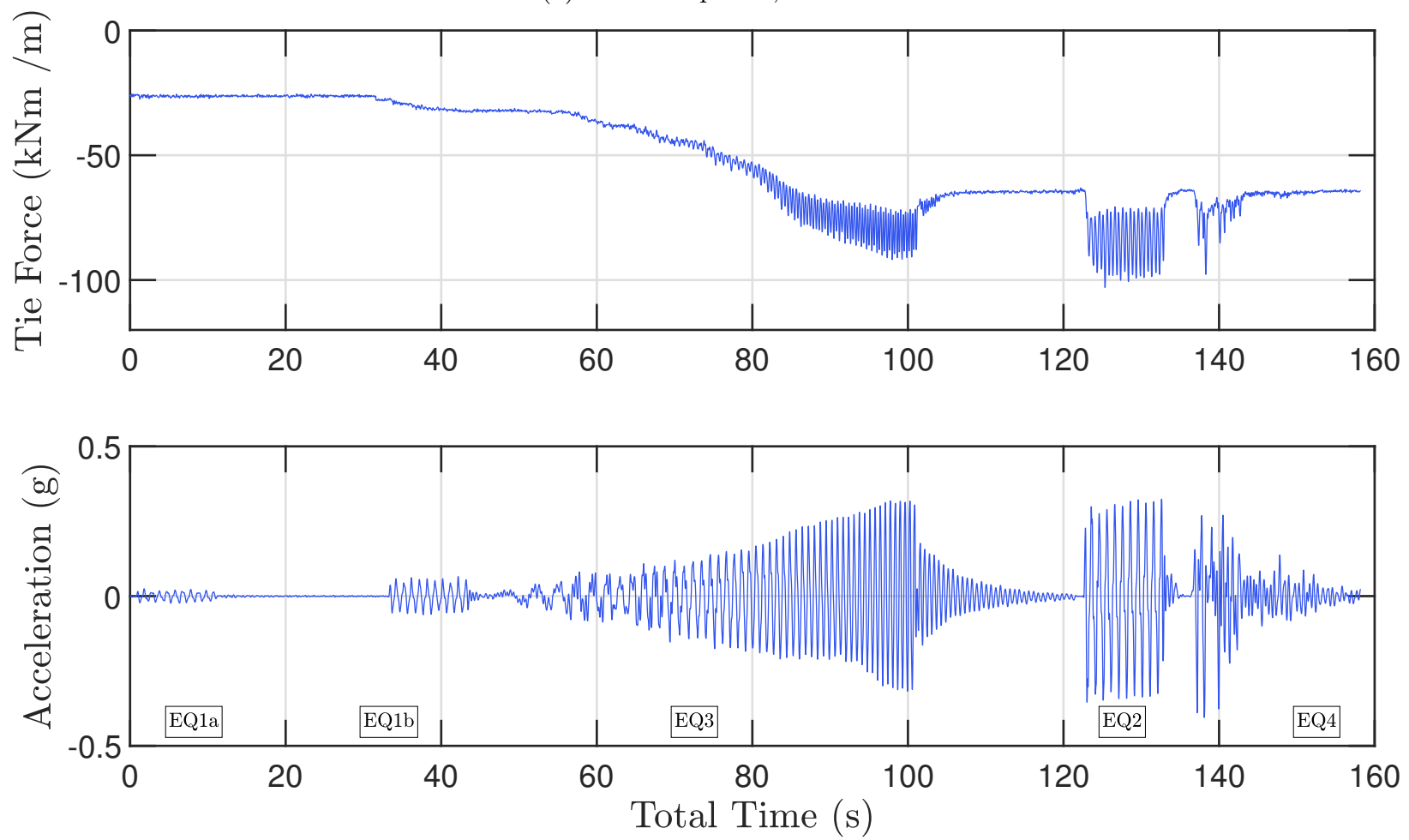

(b) All earthquakes, DS666

Figure 3: Generation of tie forces over multiple earthquakes 

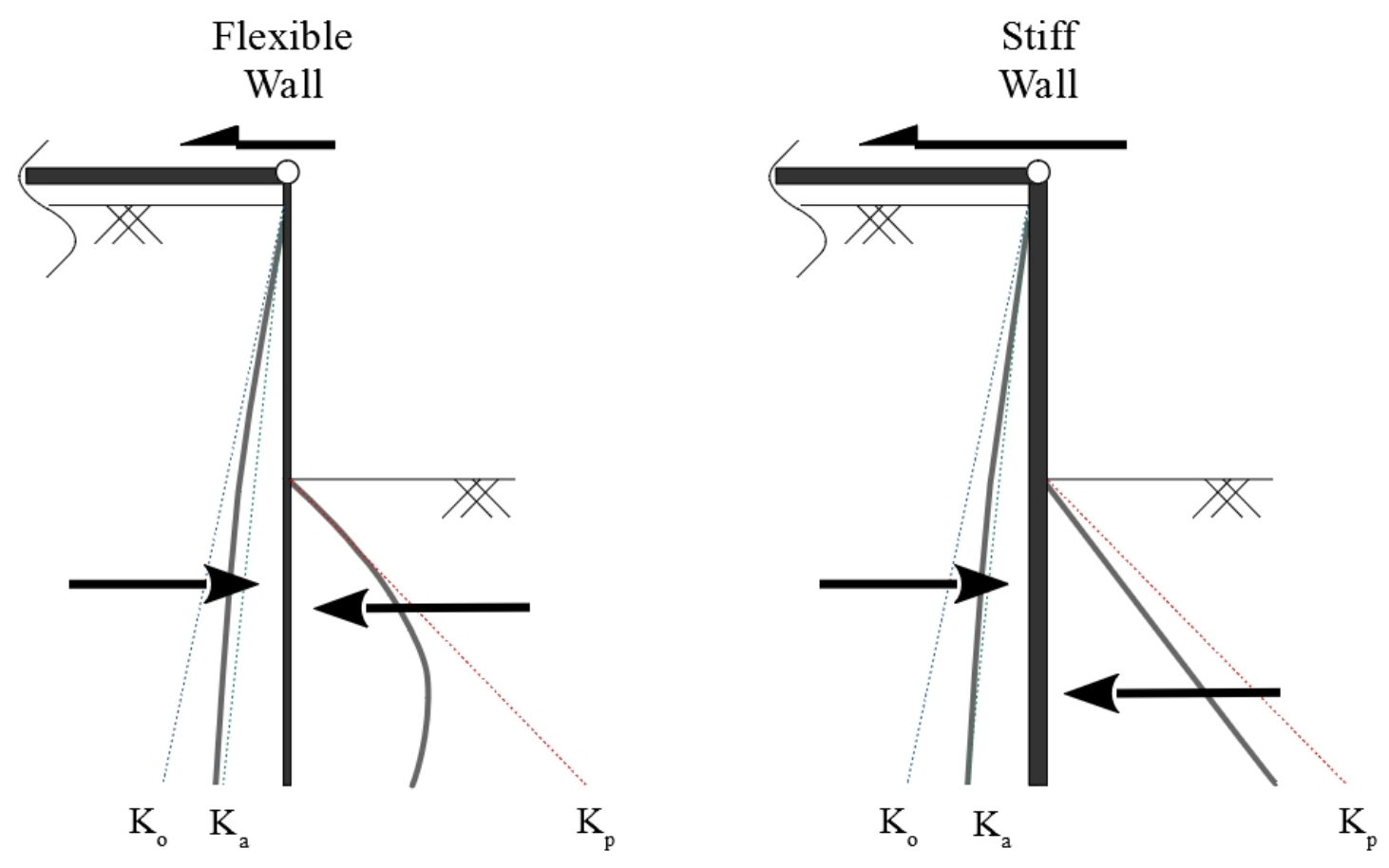

Figure 4: Dependence of tie force on the wall flexibility 


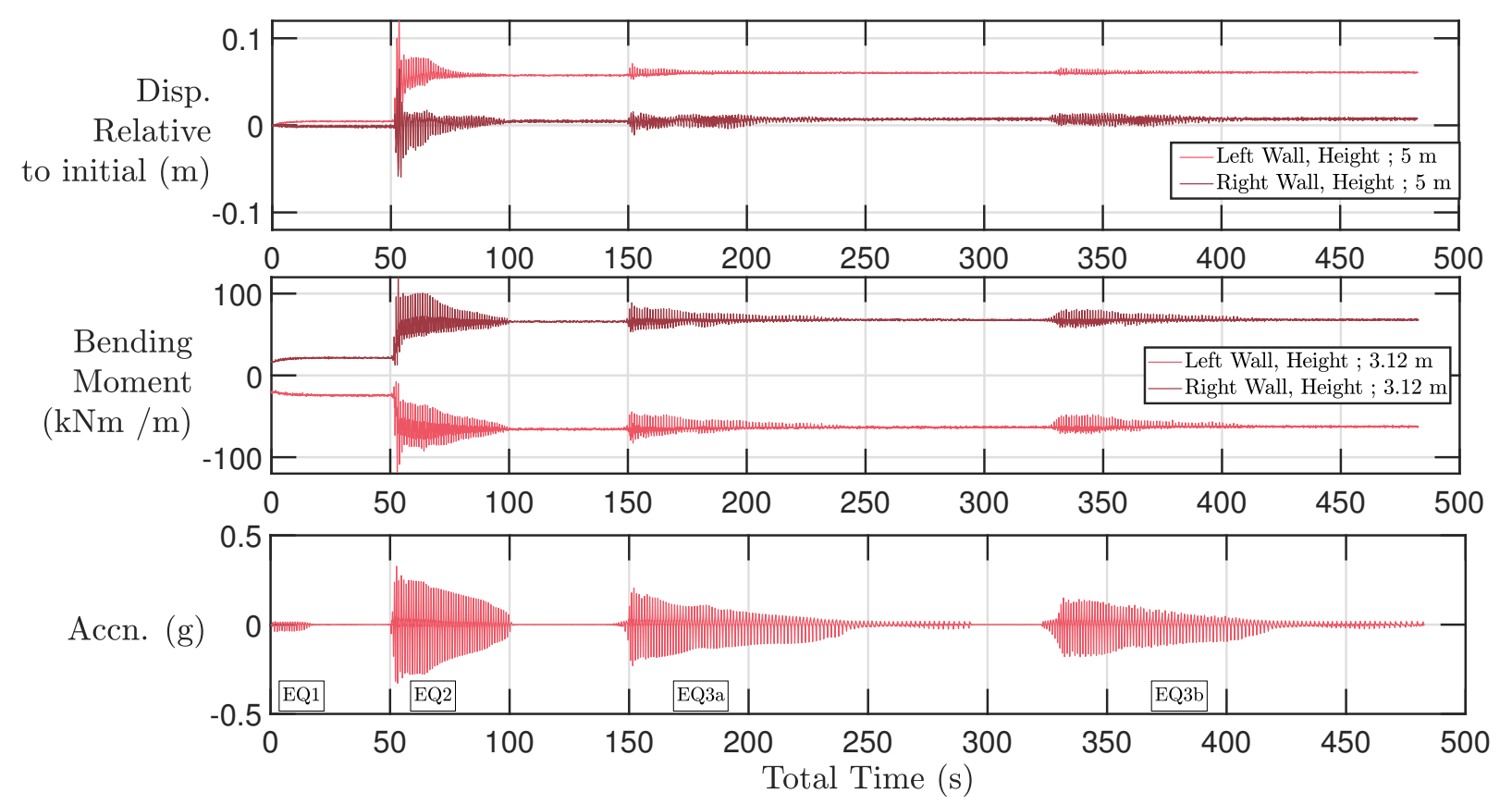

(a) All earthquakes, DF666
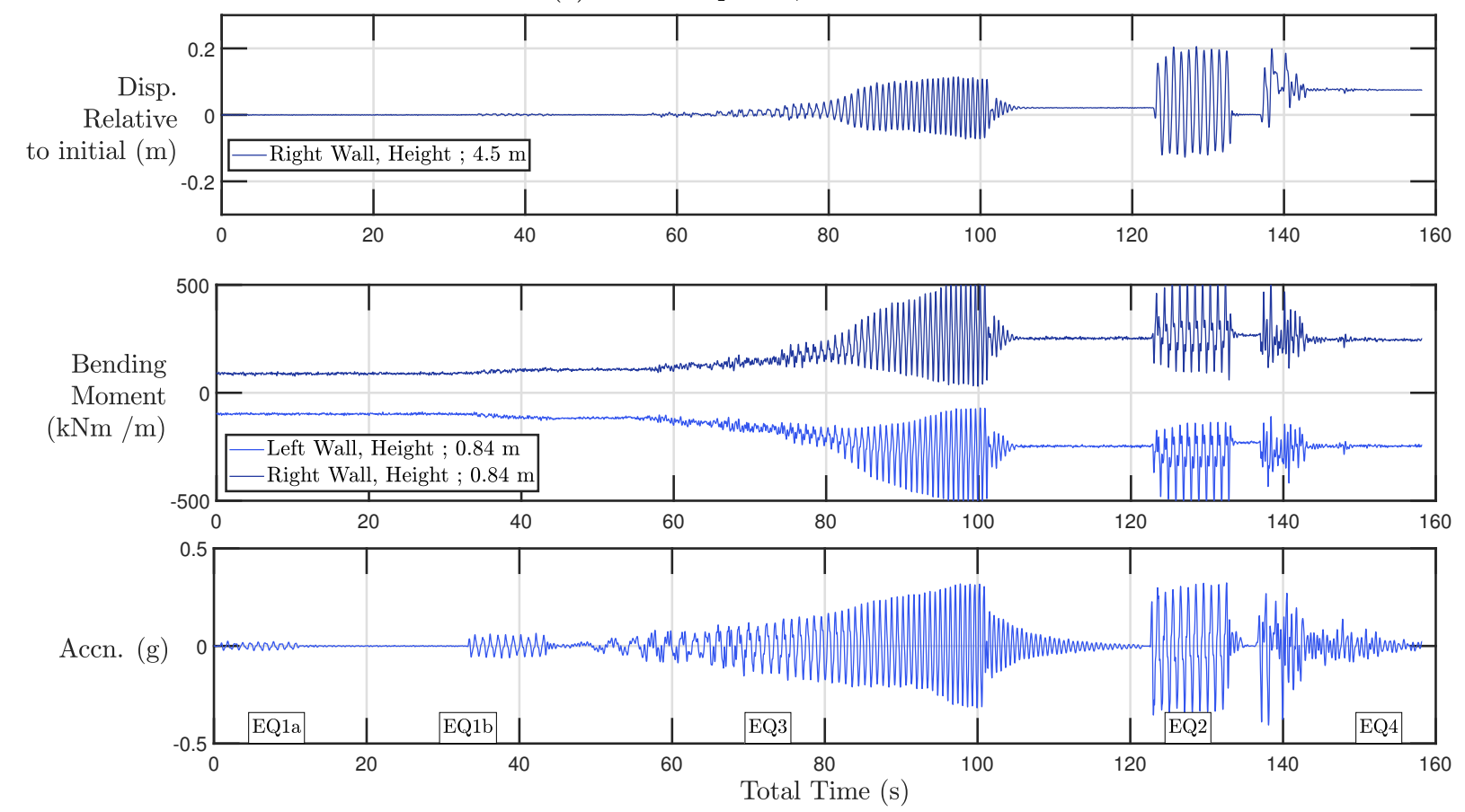

(b) All earthquakes, DS666

Figure 5: Generation of displacements and bending moments over multiple earthquakes 

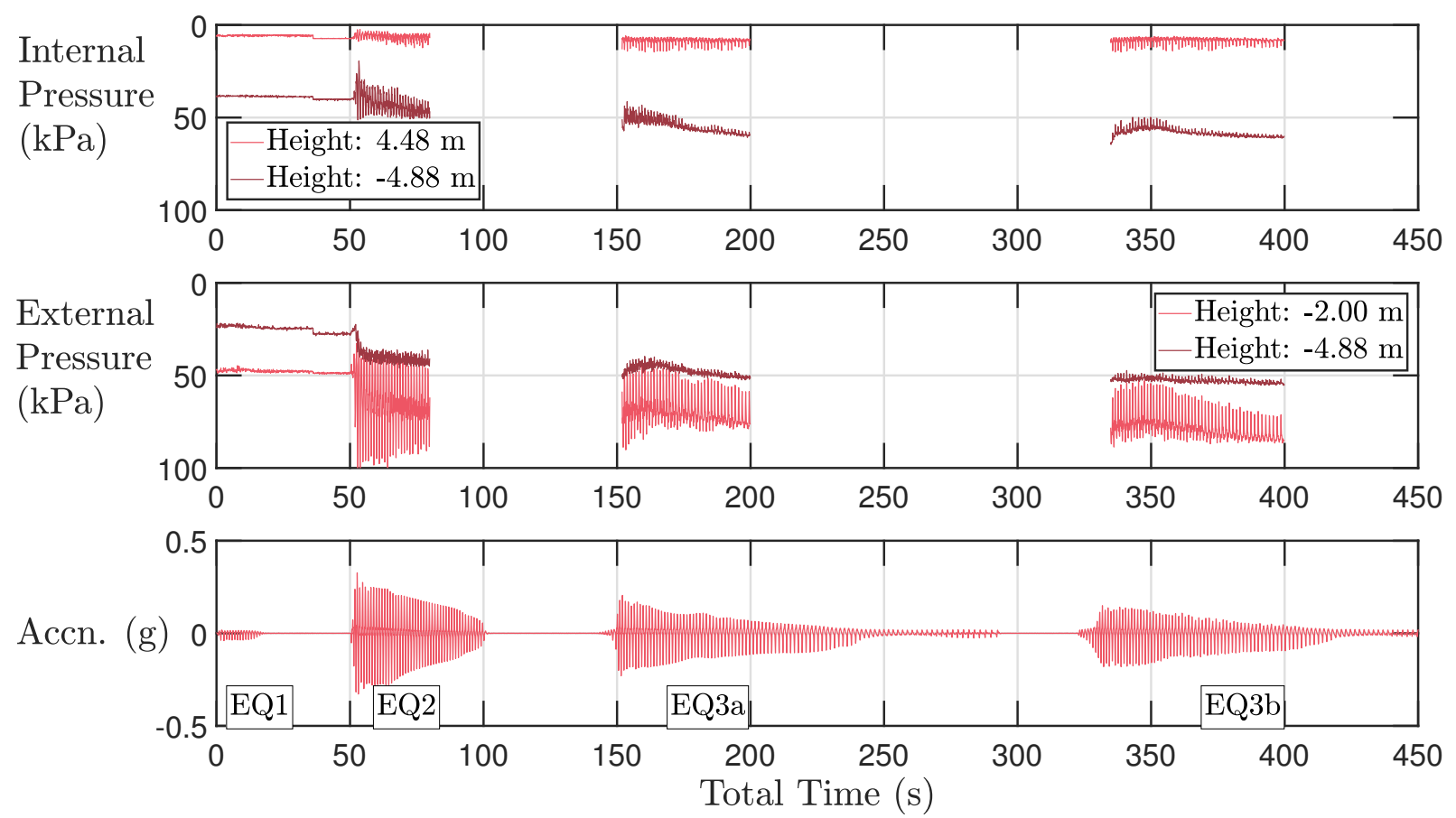

(a) All earthquakes, DF666
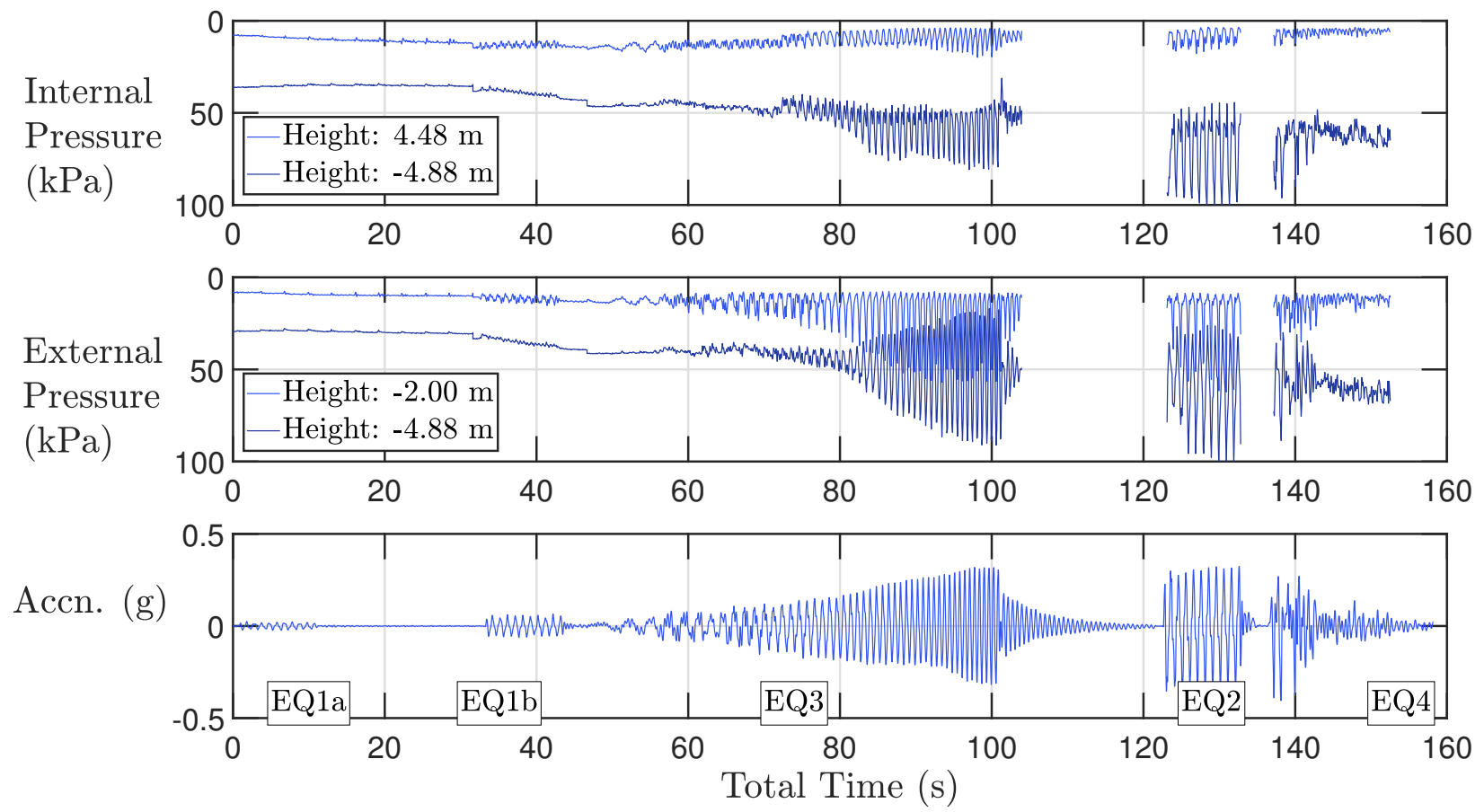

(b) All earthquakes, DS666

Figure 6: Generation of soil pressures over multiple earthquakes 


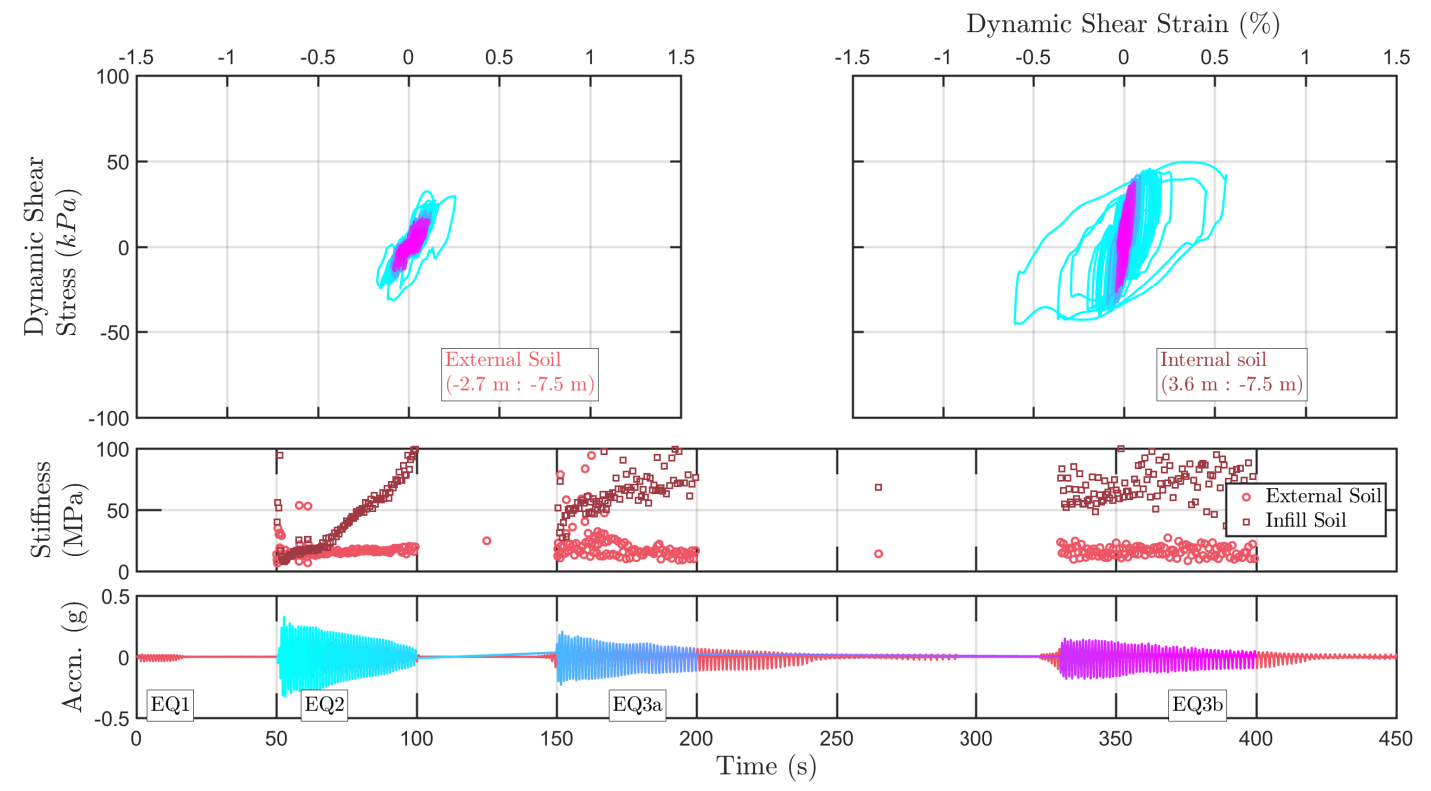

(a) All earthquakes, DF666

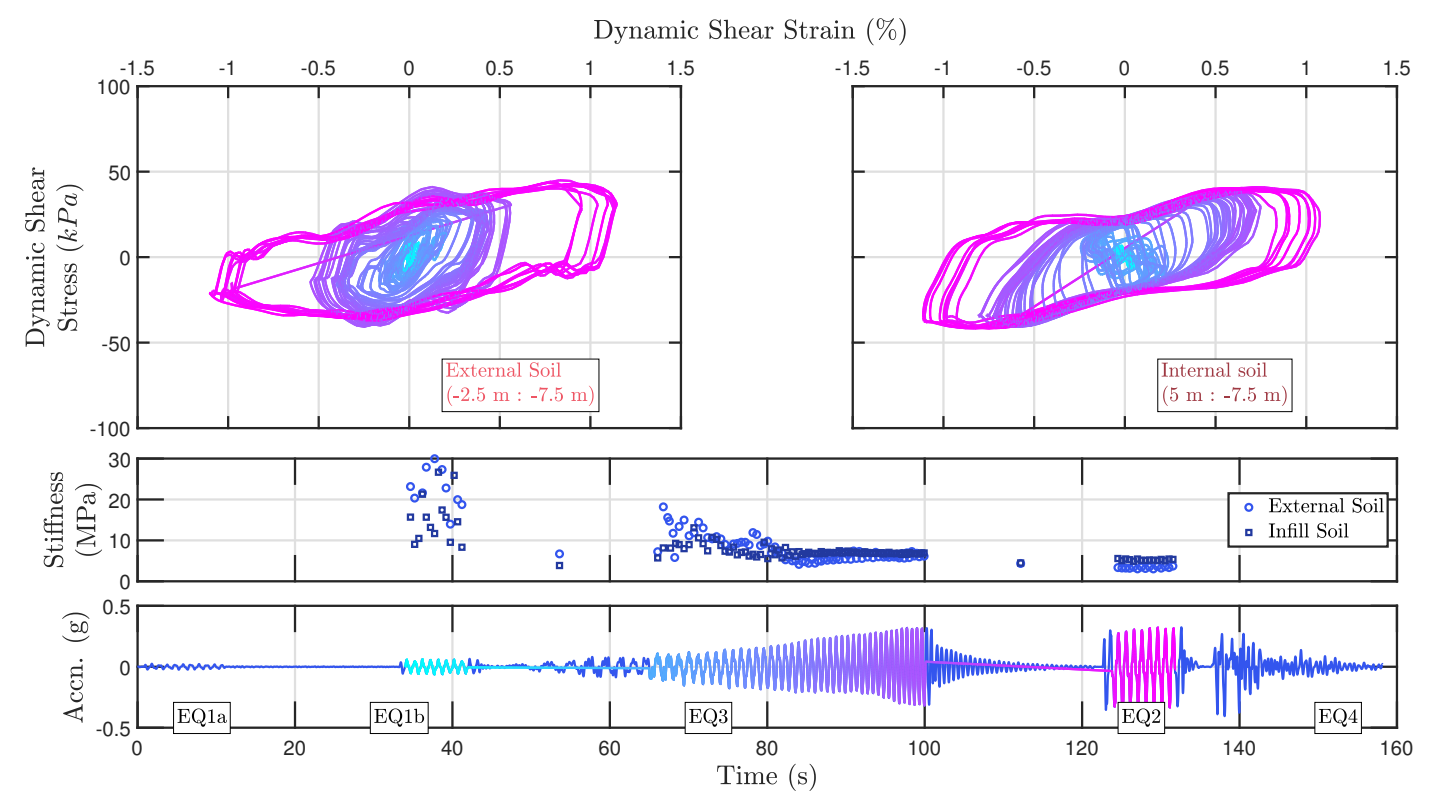

(b) All earthquakes, DS666

Figure 7: Dynamic shear stress-strain plots over multiple earthquakes 


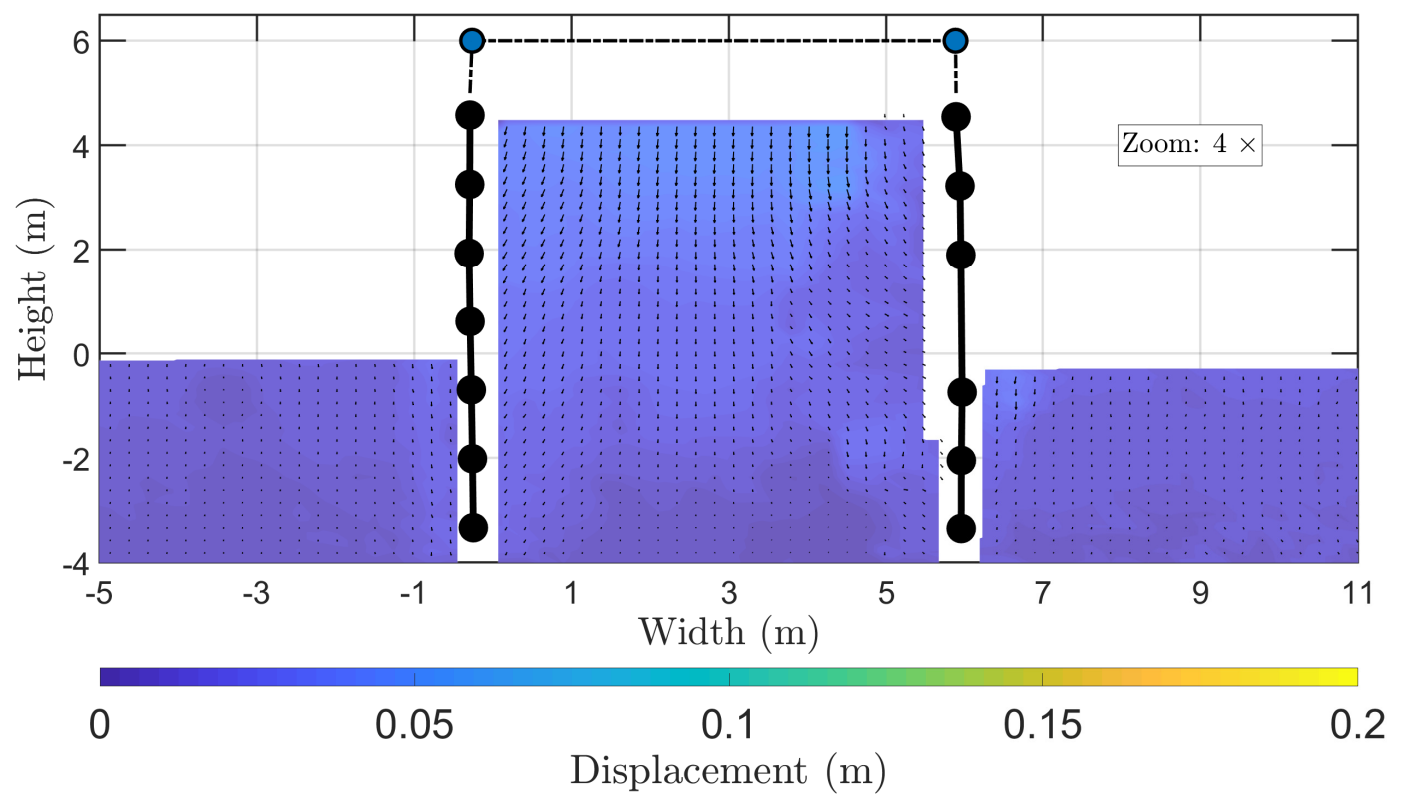

(a) Post swing up

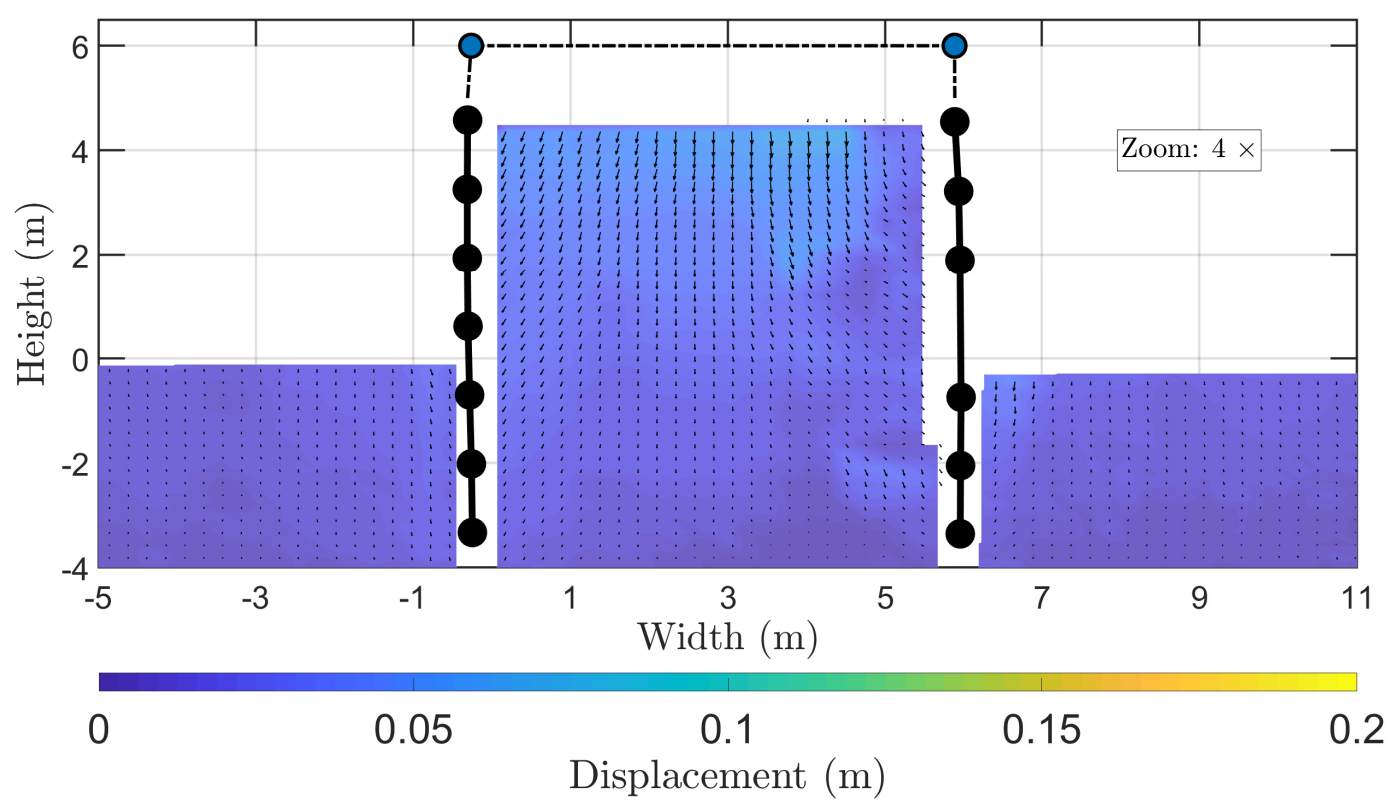

(b) Post EQ1 


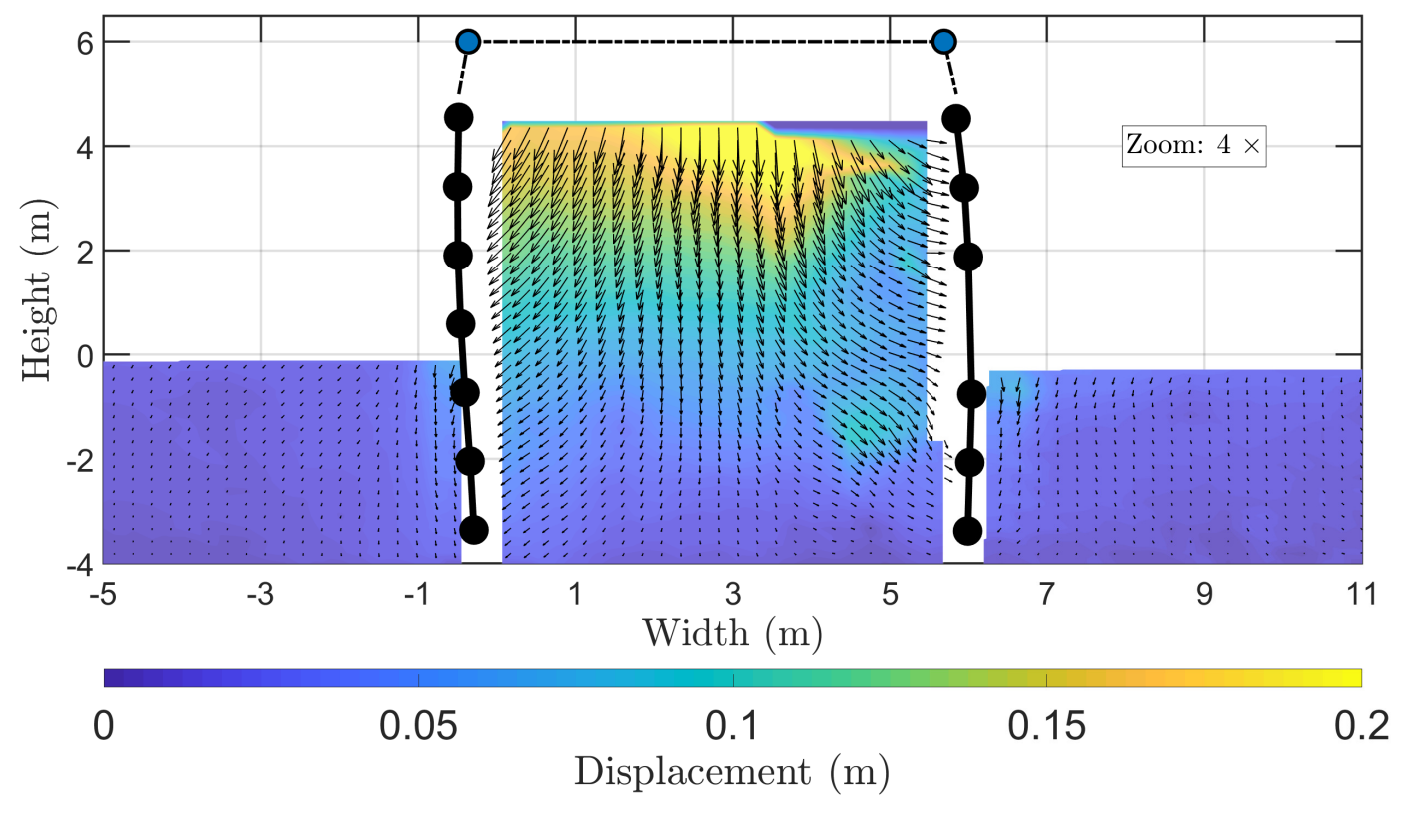

(c) Post EQ2

Figure 8: Generation of wall and soil displacements from PIV 

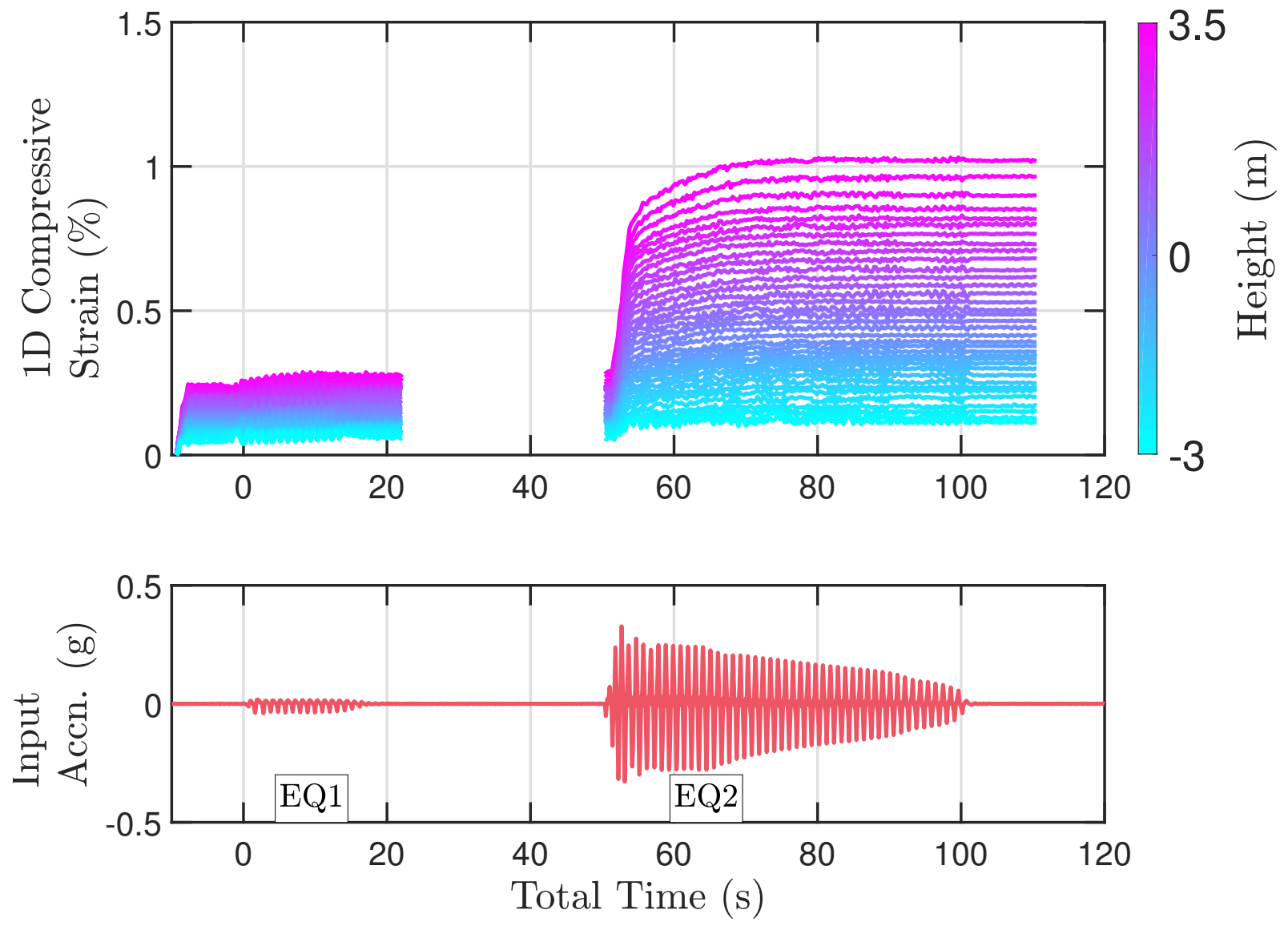

Figure 9: Estimate of strain at infill mid-line 

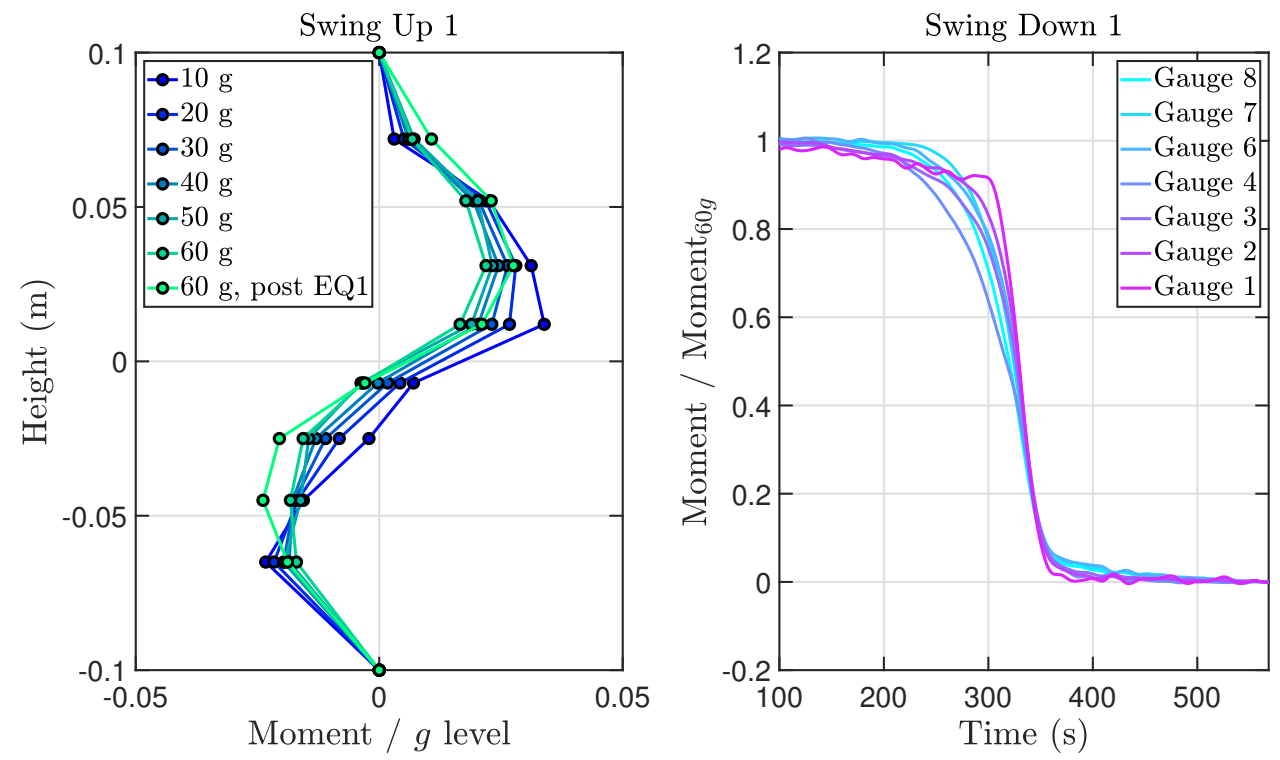

(a) Model scale bending of the right wall during swing up and down
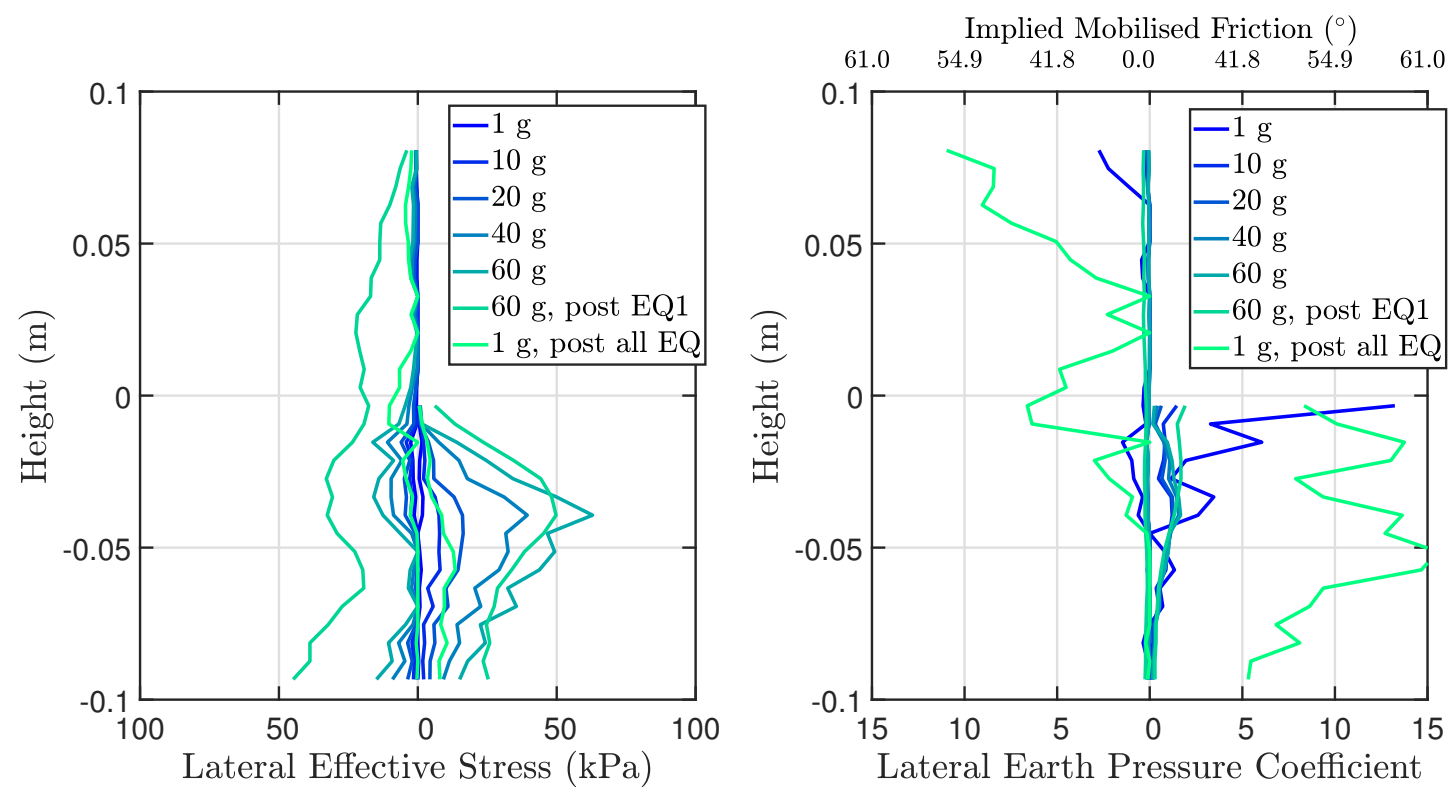

(b) Mobilised earth pressures during swing up and swing down

Figure 10: Dissimilarity of wall behaviour during swing up and swing down, DF666 


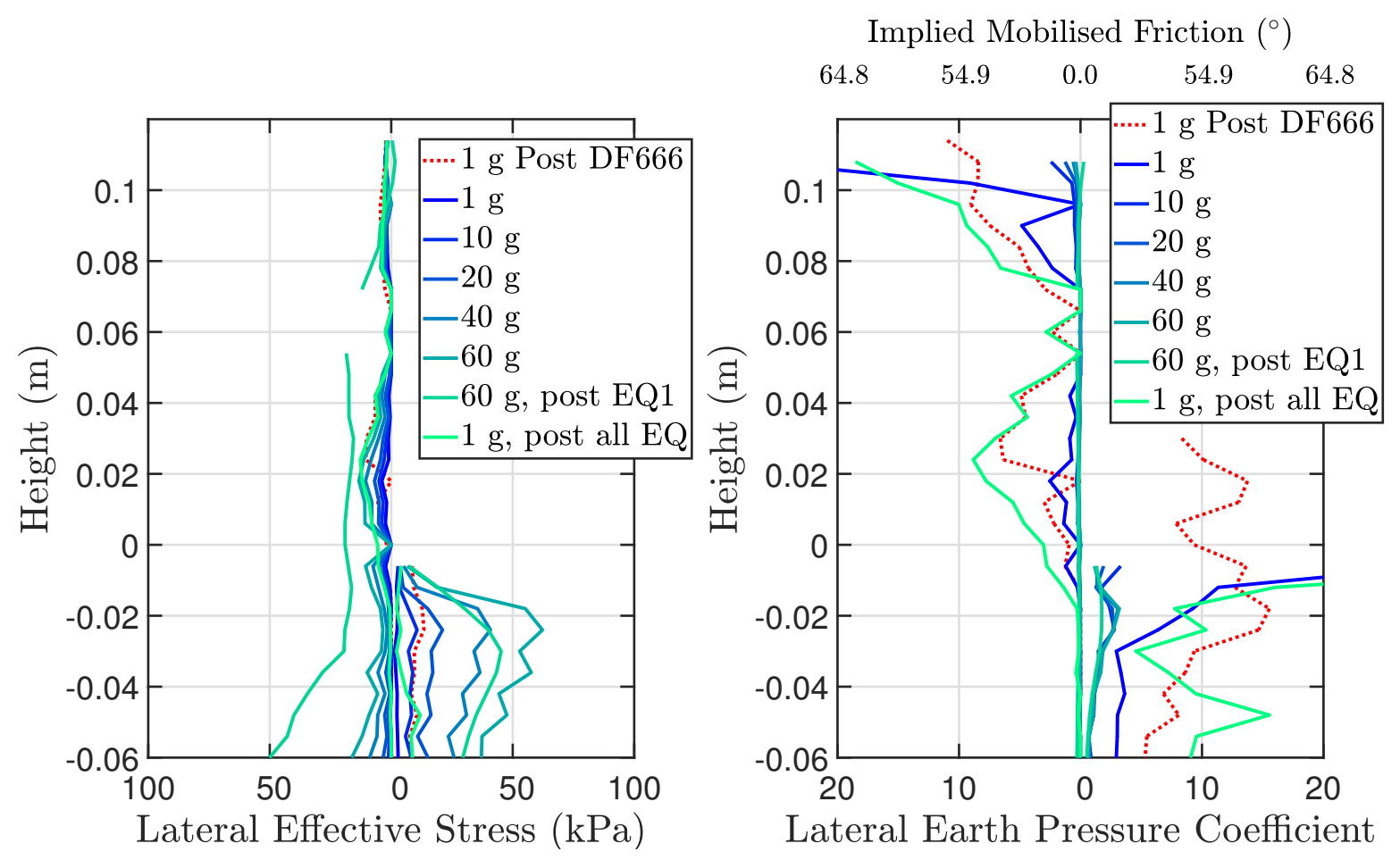

Figure 11: Earth pressure development in a previously shaken but subsequently hoovered model, DF846 


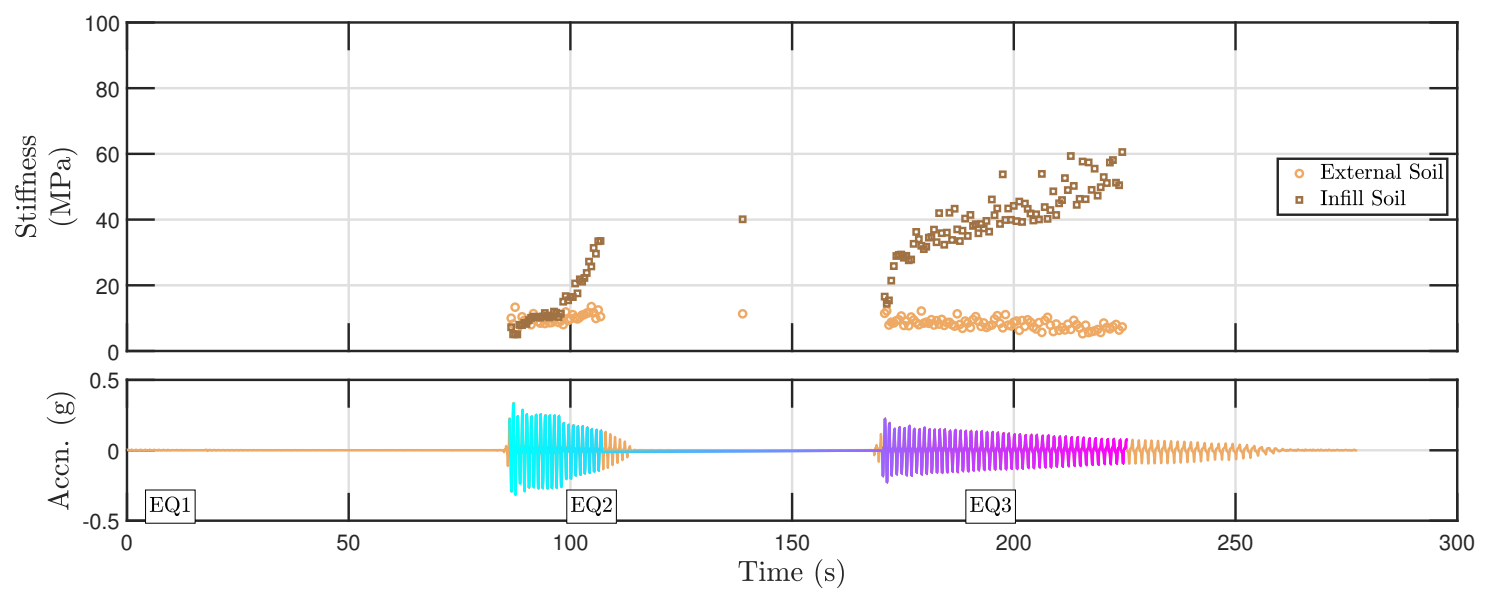

(a) Test DF846

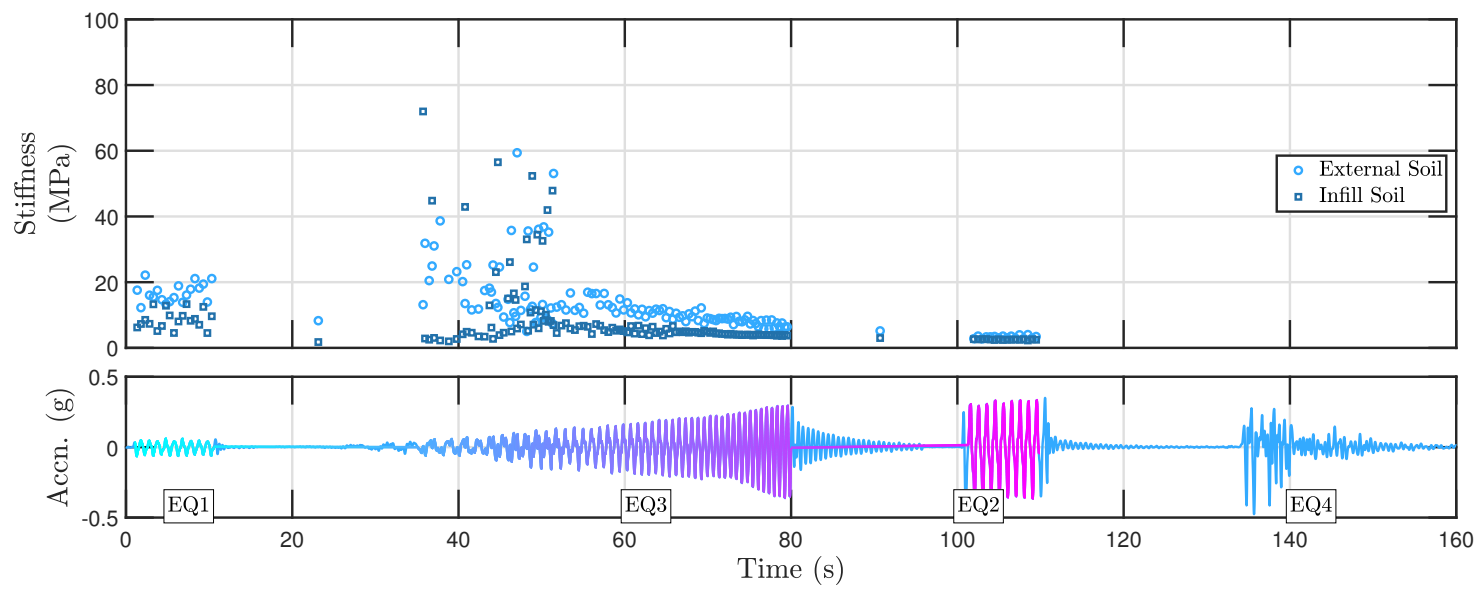

(b) Test DS846

Figure 12: Inferred average stiffness in a second centrifuge flight from dynamic stress strain loops 


\section{List of Tables}

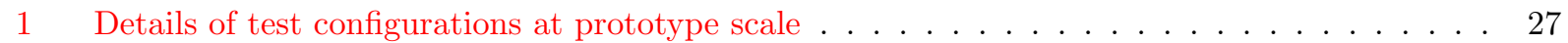

2 Details of Earthquakes applied and sequencing $\ldots \ldots \ldots \ldots \ldots$ 
Table 1: Details of test configurations at prototype scale

\begin{tabular}{llll}
\hline \multirow{2}{*}{ Variable } & \multicolumn{2}{l}{ Value } & \multirow{2}{*}{ Unit } \\
\cline { 2 - 3 } & DF666 & DF846 & \\
\hline Retained height & 6 & 8 & $(\mathrm{~m})$ \\
Embedment depth & 6 & 4 & $(\mathrm{~m})$ \\
Wall thickness & 0.18 & & $(\mathrm{~m})$ \\
Bending stiffness & 34 & & $\left(\mathrm{MNm}^{2} / \mathrm{m}\right)$ \\
Tie length & 6 & & $(\mathrm{~m})$ \\
\hline & $\mathrm{DS666}$ & $\mathrm{DS} 846$ & \\
\hline Retained height & 6 & 8 & $(\mathrm{~m})$ \\
Embedment depth & 6 & 4 & $(\mathrm{~m})$ \\
Wall thickness & 0.36 & & $(\mathrm{~m})$ \\
Bending stiffness & 272 & & $\left(\mathrm{MNm}{ }^{2} / \mathrm{m}\right)$ \\
Tie length & 6 & & $(\mathrm{~m})$ \\
\hline
\end{tabular}


Table 2: Details of Earthquakes applied and sequencing

\begin{tabular}{ccccc}
\hline & & \multicolumn{2}{c}{ Earthquake Type and identifier } & \\
Test Identifier & Small 1 Hz Sine & Large 1 Hz Sine & Scaled Kobe (1995) & Sine Sweep \\
& EQ1 & EQ2 & EQ3 & EQ4 \\
\hline DF666 & $1^{\text {st }}, 0.03,18$ & $2^{\text {nd }}, 0.35,54$ & - & $3^{\text {rd }}, 0.30,1.00-0.50$ \\
DF846 & $1^{\text {st }}, 0.03,15$ & $2^{\text {nd }}, 0.35,30$ & - & $3^{\text {rd }}, 0.30,1.20-0.50$ \\
DS666 & $1^{\text {st }}, 0.05,11$ & $3^{\text {rd }}, 0.40,11$ & $4^{\text {th }}, 0.6,25$ & $2^{\text {nd }}, 0.30,0.42-1.69$ \\
DS846 & $1^{\text {st }}, 0.06,11$ & $3^{\text {rd }}, 0.42,11$ & $4^{\text {th }}, 0.6,25$ & $2^{\text {nd }}, 0.37,0.40-1.69$ \\
\hline
\end{tabular}

Format: Sequence No., PGA $(g)$ and Duration $(s)$ or Fundamental Frequency Range $(H z)$. 